Revue d'histoire de l'Amérique française

REVUE D.HISTOIRE DE L'AMÉRIQUE FRANÇAISE

\title{
Les Patriotes de 1837-1838 d'après les documents J.-J. Girouard
}

\section{P.-A. Linteau}

Volume 21, numéro 2, septembre 1967

URI : https://id.erudit.org/iderudit/302673ar

DOI : https://doi.org/10.7202/302673ar

Aller au sommaire du numéro

Éditeur(s)

Institut d'histoire de l'Amérique française

ISSN

0035-2357 (imprimé)

1492-1383 (numérique)

Découvrir la revue

Citer ce document

Linteau, P.-A. (1967). Les Patriotes de 1837-1838 d'après les documents J.-J.

Girouard. Revue d'histoire de l'Amérique française, 21(2), 281-311.

https://doi.org/10.7202/302673ar d'utilisation que vous pouvez consulter en ligne.

https://apropos.erudit.org/fr/usagers/politique-dutilisation/ 


\section{DOCUMENTS INÉDITS}

\section{LES PATRIOTES DE 1837-1838 D'ĀPRES LES DOCUMENTS J.-J. GIROUARD}

La célébration d'un anniversaire, qu'il soit heureux ou malheureux, a toujours le mérite de rappeler aux hommes les événements du passé et de les inviter à y réfléchir. Pour l'historien, c'est une occasion toute choisie de remettre en lumière certains faits du passé trop souvent oubliés et parfois de les présenter sous un éclairage nouveau. C'est le cas, cette année, du $130^{\circ}$ anniversaire de la Rébellion de 1837-1838. La Revue d'histoire de l'Amérique française commémore à sa façon cet événement en publiant certains textes peu connus du grand public.*

L'Institut d'histoire de l'Amérique française possède en effet d'intéressants documents ayant appartenu à l'un des Patriotes, Jean-Joseph Girouard, et qui ont été remis à l'Institut par son arrière-petite-fille, Mme Jeanne Girouard-Décarie.

Rappelons brièvement que J.-J. Girouard, né à Québec le 11 novembre 1795, est décédé à St-Benoît le 18 septembre 1855. C'est à ce dernier endroit qu'il passa la majeure partie de sa vie, y exerçant la profession de notaire. Il fut député du comté des Deux-Montagnes à la Chambre d'Assemblée du Bas-Canada de 1831 à 1838. Personnage influent chez les Patriotes, sa tête fut mise à prix en décembre 1837. Il fut emprisonné de décembre 1837 à juillet 1838 et de novembre à décembre $1838 .{ }^{1}$

Les papiers de J.-J. Girouard sont extrêmement intéressants pour l'étude de la Rébellion de 1837-1838. Notre Revue a déjà publié quatre lettres de Girouard à sa femme, écrites de la prison de Montréal, et deux lettres de Louis-Joseph Papineau à Girouard. ${ }^{2}$ Le document qui retient notre attention à l'occasion du $130^{\circ}$ anniversaire de la Rébellion est un manuscrit de 30

* C'est pour cette raison que nous devons reporter à un numéro ultérieur la correspondance de sir Joseph Dubuc, dont une partie a paru dans les nos précédents.

1 Aegidius Fauteux, Patriotes de 1837-1838 (Montréal, Les Editions des Dix, 1950), 253-256. 463-464.

2 Voir RHAF, VII : 110-112; XV : 283-288; XIX: 127-128, 302-303, 
pages qui, à notre connaissance, n'a pas encore été publié en entier.

Ce texte comprend:

1 - La liste des 495 Patriotes détenus à Montréal et leurs qualités: âge, domicile, profession, etc...., suivie d'une "Récapitulation". (Ces renseignements seront précieux pour ceux qui s'intéressent à l'histoire sociale de la Rébellion.)

2 - La liste des Patriotes emprisonnés à Québec et leurs qualités.

3 - La liste des Patriotes incarcérés à Trois-Rivières.

4 - La liste des Patriotes qui ont dû s'enfuir ou se cacher.

5 - La liste des citoyens dont la tête fut mise à prix.

6 - Des observations générales sur la Rébellion.

7 - Des notes concernant certains des Patriotes mentionnés dans la première liste.

Le document a été rédigé le ler juin 1838. Dans ses observations, l'auteur de la compilation note qu'il n'a pas eu accès aux documents officiels et s'excuse des erreurs minimes qui ont pu se glisser dans son travail.

En terminant souhaitons que l'inventaire, en cours de réalisation, des papiers de J.-J. Girouard déposés aux archives de l'Institut d'histoire de l'Amérique française apporte des renseignements nouveaux à l'étude du deuxième quart du XIX $^{\circ}$ siècle.

P.-A. Linteau 


\section{TA B L E A U}

des Patriotes qui ont été incarcérés dans les prisons de Montréal, sous prévention de haute trahison à la fin de 1837 et au commencement de 1838 .

\begin{tabular}{|c|c|c|c|c|c|c|c|c|c|c|c|}
\hline $\begin{array}{c}\text { NOMS } \\
\text { des détenus }\end{array}$ & AGE & $\begin{array}{l}\text { PROFESSIONS } \\
\text { et autres } \\
\text { qualifications }\end{array}$ & DEMEURES & $\begin{array}{c}\text { DATI } \\
\text { de leu } \\
\text { arrestati } \\
\text { ou } \\
\text { empriso } \\
\text { nemen }\end{array}$ & & $\begin{array}{c}\begin{array}{c}\text { Prisonni } \\
\text { qui ont } \\
\text { élargi }\end{array} \\
\begin{array}{r}\text { Date de } \\
\text { sortie }\end{array}\end{array}$ & & $\begin{array}{c}\text { sans } \\
\text { caution }\end{array}$ & $\begin{array}{l}\text { sous } \\
\text { caution }\end{array}$ & $\begin{array}{c}\text { Nombre de } \\
\text { jours do } \\
\text { détention, } \\
\text { au 1er } \\
\text { Juin } 1838\end{array}$ & $\begin{array}{c}\text { Indication } \\
\text { des } \\
\text { notes }\end{array}$ \\
\hline $\begin{array}{l}\text { Autier, Constant } \\
\text { Arquet, Michel } \\
\text { Auger, Luc } \\
\text { Aubry, Abraham } \\
\text { Arbick, Basile } \\
\text { Aubry, François } \\
\text { Aubain, } \\
\text { Aubry, Michel } \\
\text { Anderson, John } \\
\text { Ausbrou, Thomas } \\
\text { Amiot, Pierre } \\
\text { Arcan, Olivier } \\
\text { Arcan, J. Bte }\end{array}$ & $\begin{array}{l}18 \\
50 \\
40 \\
30 \\
25 \\
37 \\
44 \\
28\end{array}$ & $\begin{array}{l}\text { Cultivateur } \\
\text { Journalier } \\
\text { Cultivateur } \\
\text { “ } \\
\text { Journalier } \\
\text { Menuisier } \\
\text { Cultivateur }\end{array}$ & $\begin{array}{c}\text { St Hilaire } \\
\text { St Valentin } \\
\text { St-Benoit } \\
\text { St Eustache } \\
\text { St Benoit } \\
\text { St Eustache } \\
\text { “" }\end{array}$ & $\begin{array}{c}\text { Novre } \\
\text { Decre } \\
\text { "“ } \\
\text { “" } \\
\text { "“ } \\
\text { “" } \\
\text { Janv. } \\
\text { Dec. } \\
\text { Mars } \\
\text { Dec. }\end{array}$ & $\begin{array}{r}30 \\
9 \\
16 \\
14 \\
15 \\
14 \\
6 \\
16 \\
20 \\
29 \\
8 \\
28 \\
9\end{array}$ & $\begin{array}{l}\text { Decre } \\
\text { Mars } \\
\text { Decre } \\
\text { Mars } \\
\text { Decre } \\
\text { Mars }\end{array}$ & $\begin{array}{l}28 \\
18 \\
13 \\
18 \\
13\end{array}$ & 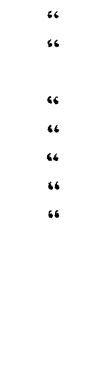 & & & \\
\hline $\begin{array}{l}\text { Boucherville, George de } \\
\text { Bardy, A.E. } \\
\text { Bougrette, Joseph } \\
\text { Blanchet, Augn } \\
\text { Beauvouloir, Tousst. } \\
\text { Beaulac, Felix } \\
\text { Bellemare, Joseph } \\
\text { Bachamp, Francois } \\
\text { Barsalou, Antoine } \\
\text { Bourdon, Louis } \\
\text { Beaulac, Pierre } \\
\text { Bourgeois, " }\end{array}$ & $\begin{array}{l}20 \\
40 \\
36 \\
16 \\
21 \\
41 \\
58\end{array}$ & $\begin{array}{l}\text { Montréal (sic) } \\
\text { N.P. } \\
\text { Cultivateur } \\
\text { Journalier } \\
\text { Cultivateur } \\
\text { “" } \\
\text { Marchand } \\
\text { Aubergiste }\end{array}$ & $\begin{array}{l}\text { Avocat (sic) } \\
\text { St Athanase } \\
\text { Présentation } \\
\quad \text { “" } \\
\text { St Charles } \\
\text { "“ } \\
\text { St Jean Baptiste } \\
\text { St Césaire } \\
\text { St Ours } \\
\text { St Denis }\end{array}$ & 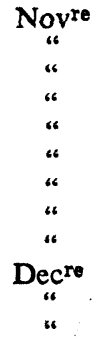 & $\begin{array}{r}16 \\
24 \\
30 \\
30 \\
“ \\
“ \\
“ \\
7 \\
24 \\
9\end{array}$ & $\begin{array}{c}\text { Decre } \\
“ \\
“ \\
“ \\
“ \\
\text { “ } \\
\text { Fev. } \\
\text { Mai }\end{array}$ & $\begin{array}{l}27 \\
11 \\
16 \\
4 \\
\text { “ } \\
\text { “ }\end{array}$ & 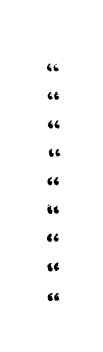 & & . & $\begin{array}{l}\text { Voyez la } \\
\text { note (a) }\end{array}$ \\
\hline
\end{tabular}




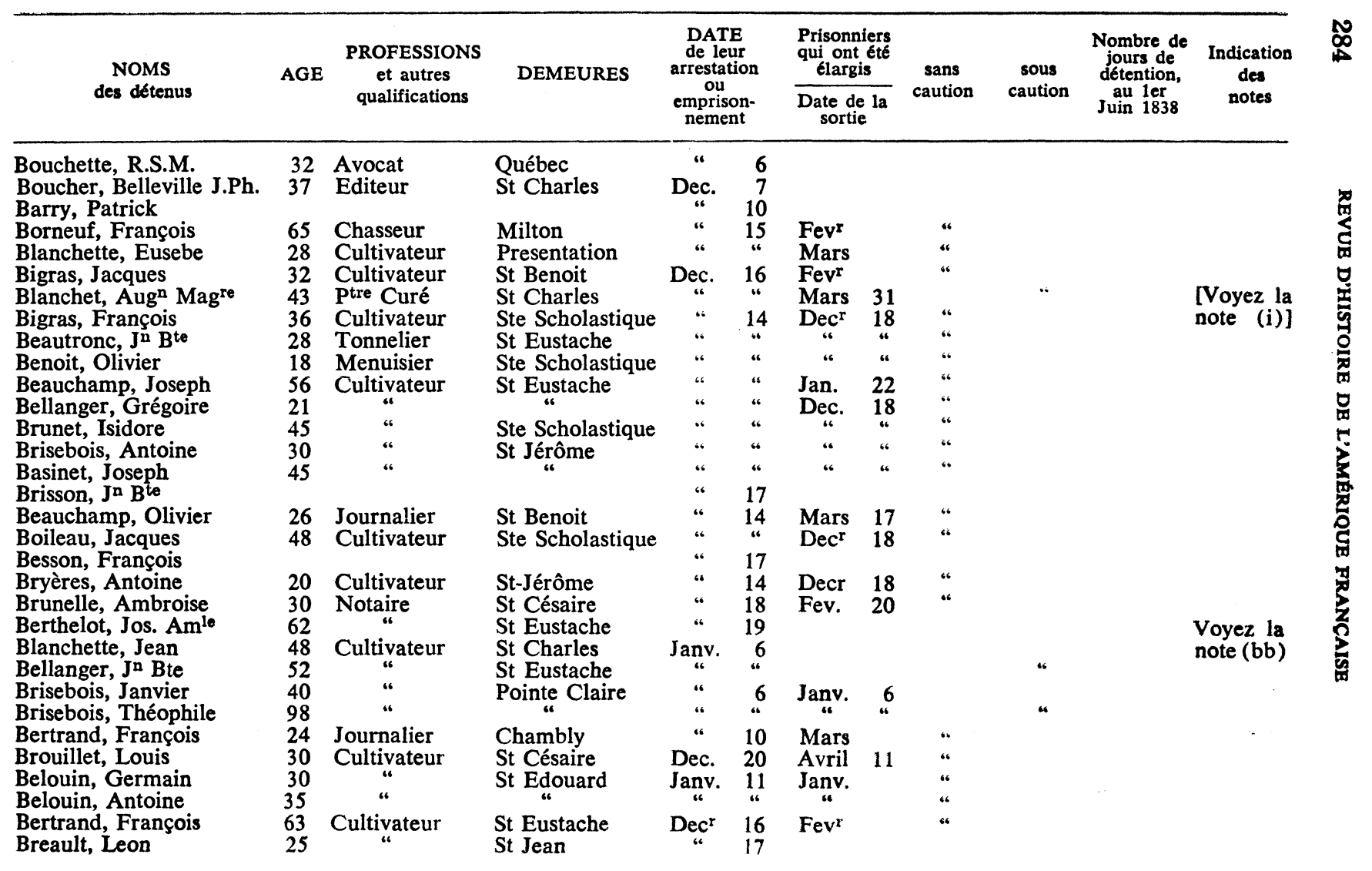




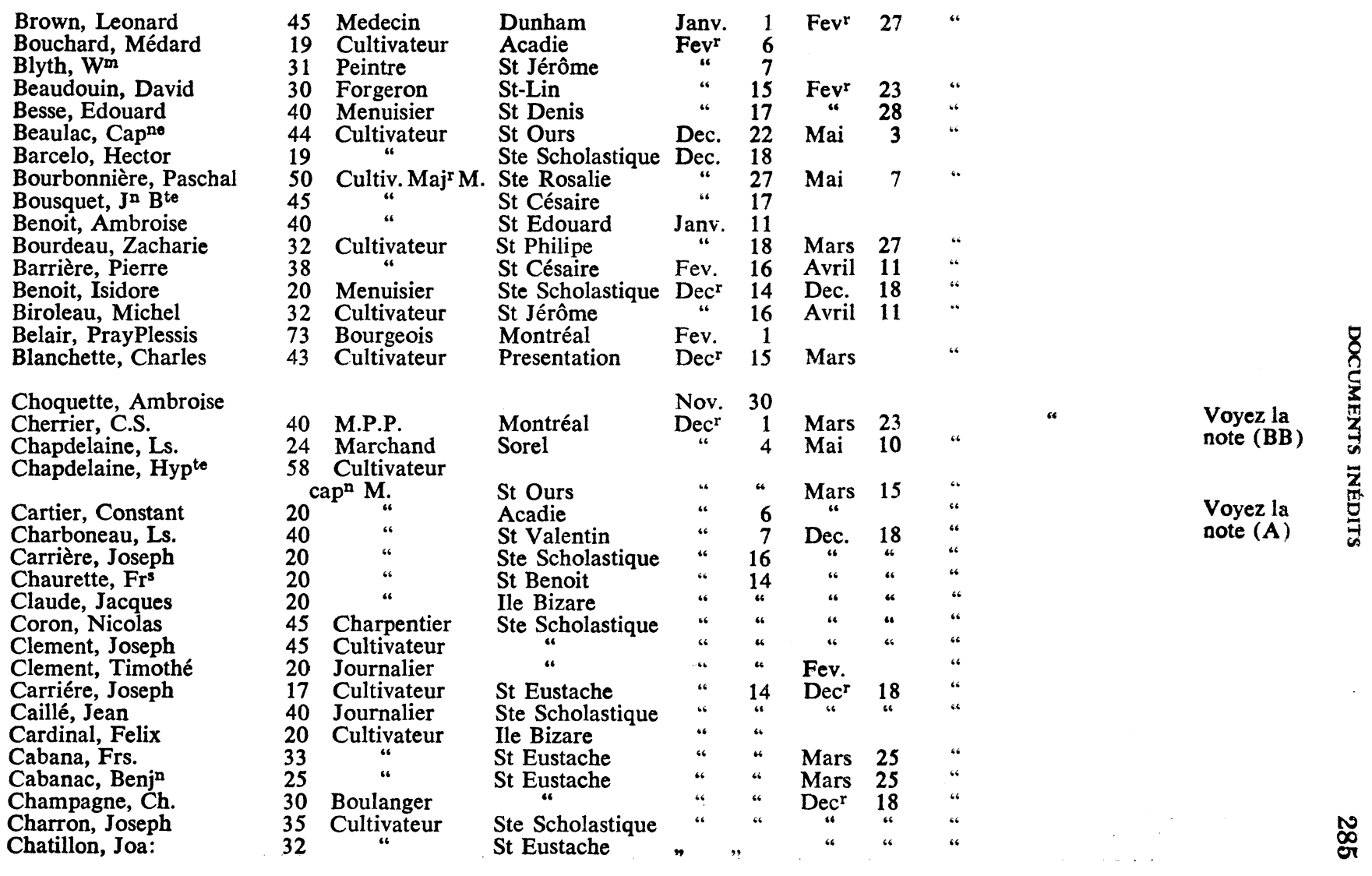




\begin{tabular}{|c|c|c|c|c|c|c|c|c|c|c|c|}
\hline \multirow{19}{*}{$\begin{array}{l}\text { Chartrand, J. Bte } \\
\text { Coursolle; Ls } \\
\text { Chaperon, J. Bte sen } \\
\text { Chaperon, J.B. jun } \\
\text { Chaperon, Pre } \\
\text { Chicot, J.B. } \\
\text { Chisholm, Robt } \\
\text { Chaurette, Ls. } \\
\text { Callaghan, Peter } \\
\text { Carrière, Eustache } \\
\text { Charlebois, Léon } \\
\text { Charlebois, Hya. } \\
\text { Carrière, Michel } \\
\text { Consigny, Antoine } \\
\text { Chesnier, Felix } \\
\text { Charland, Domque } \\
\text { Crochetière, Stanislas } \\
\text { Chevalier, Pierre } \\
\text { Casavan, Pierre } \\
\text { Campbell, Marc } \\
\text { Caillé, Jacques } \\
\text { Cardau, Marcel }\end{array}$} & \multirow{2}{*}{$\frac{\text { AGE }}{30}$} & \multirow{2}{*}{$\begin{array}{c}\begin{array}{c}\text { PROFESSIONS } \\
\text { et autres } \\
\text { qualifications }\end{array} \\
\text { Voyageur }\end{array}$} & DEMEURES & \multicolumn{2}{|c|}{$\begin{array}{c}\text { DATE } \\
\text { de leur } \\
\text { arrestation } \\
\text { ou } \\
\text { emprison- } \\
\text { nement }\end{array}$} & \multicolumn{2}{|c|}{$\begin{array}{c}\begin{array}{c}\text { Prisonniers } \\
\text { qui ont été } \\
\text { élargis }\end{array} \\
\begin{array}{c}\text { Date de la } \\
\text { sortie. }\end{array}\end{array}$} & \multirow{2}{*}{$\begin{array}{c}\begin{array}{c}\text { sans } \\
\text { caution }\end{array} \\
\text { “ }\end{array}$} & \multirow[t]{2}{*}{$\begin{array}{l}\text { sous } \\
\text { caution }\end{array}$} & \multirow[t]{2}{*}{$\begin{array}{c}\text { Nombre de } \\
\text { jours de } \\
\text { détention, } \\
\text { au ler } \\
\text { Juin } 1838\end{array}$} & \multirow[t]{2}{*}{$\begin{array}{c}\text { Indication } \\
\text { des } \\
\text { notes }\end{array}$} \\
\hline & & & “ & “ & $"$ & Mars & 25 & & & & \\
\hline & 50 & Aubergiste & St Benoit & “ & 16 & & & & & & \\
\hline & 42 & Cultivateur & St Edouard & Janv. & 11 & Fev. & & $"$ & & & \\
\hline & 20 & 6 & " & “ & “ & 6 & & “6 & & & \\
\hline & 18 & $“$ & “ & “ & $\cdots$ & “ & & $“$ & & & \\
\hline & 38 & “ & " , & “ & & “ & & $"$ & & & \\
\hline & $\begin{array}{l}23 \\
52\end{array}$ & $\begin{array}{l}\text { Forgeron } \\
\text { Marchand }\end{array}$ & $\begin{array}{l}\text { Godmanchester } \\
\text { Ste Scholastique }\end{array}$ & “" & 16 & “" & & “ & " & & \\
\hline & 34 & Instituteur & Ste Marie & Fev. & 5 & 4 & 7 & "6 & & & \\
\hline & 57 & Cultivateur & St Denis & “ & 17 & mars & 25 & & " & & \\
\hline & $\begin{array}{l}35 \\
30\end{array}$ & Aubergiste & Montréal & Mars & 1 & “ & & " & & & \\
\hline & $\begin{array}{l}30 \\
46\end{array}$ & $\begin{array}{l}\text { Notaire } \\
\text { Cultivateur }\end{array}$ & $\begin{array}{l}\text { Vaudreuil } \\
\text { St Eustache }\end{array}$ & Dec. & $\begin{array}{l}20 \\
17\end{array}$ & & & & & & \\
\hline & 23 & Médecin & St Damase & “ & 28 & & & & & & \\
\hline & 24 & Notaire & St Eustache & Janv. & 2 & & & & & & \\
\hline & 38 & Marchand & Yamaska & Mars & 28 & & & & & & \\
\hline & $\begin{array}{l}32 \\
30\end{array}$ & $\begin{array}{l}\text { Menuisier } \\
\text { Marchand }\end{array}$ & $\begin{array}{l}\text { Montréal } \\
\text { St Eustache }\end{array}$ & Dec. & $\begin{array}{r}9 \\
22\end{array}$ & $\underset{\text { mars }}{\operatorname{man}}$ & $\begin{array}{l}27 \\
27\end{array}$ & “ & & & \\
\hline & 19 & Tonnelier & Montreal & “ & 28 & $“$ & “ & “ & & & \\
\hline & 16 & Marchand & Pte aux trembles & Janv. & 26 & “ & 3 & $“$ & & & \\
\hline & $\begin{array}{l}48 \\
22\end{array}$ & $\underset{\text { Cultivateur }}{ }$ & $\begin{array}{l}\text { Ste Scholastique } \\
\text { St Denis }\end{array}$ & $\begin{array}{l}\text { Dec. } \\
\text { Fev. }\end{array}$ & $\begin{array}{l}14 \\
17\end{array}$ & $\begin{array}{l}\text { dec. } \\
\text { mars }\end{array}$ & 18 & $"$ & “ & & \\
\hline $\begin{array}{l}\text { Dubuc, Jean } \\
\text { Duquet, Frs }\end{array}$ & 40 & Aubergiste & Montréal & Nov. & $\begin{array}{l}16 \\
28\end{array}$ & Dec. & $\begin{array}{r}18 \\
2\end{array}$ & “ & & & \\
\hline Durocher, Eusèbe & 36 & Marchand & St Charles & “ & 26 & & & & & & \\
\hline Daigneau, Joseph & 40 & Cultivateur & Longueuil & “ & 30 & $" 6$ & 15 & “ & & & \\
\hline Duvert, Ls Chicou & 50 & Notaire & St Charles & “ & 25 & & & & & & \\
\hline Doran, Wm. & & & & & & Dec. & 1 & & $“$ & & \\
\hline Dorion, Jacques & & M.P.P. & St Ours & Dec. & 2 & & & & “ & & \\
\hline $\begin{array}{l}\text { Dillon, George } \\
\text { Donais, Amable }\end{array}$ & 27 & Marchand & Montréal & “ & 3 & Mars & 23 & “" & & & \\
\hline Donais, Amable & 22 & Journaller & St Cyprien & & 7 & & & & & & \\
\hline
\end{tabular}


Desrivières, Rodolphe Dragon, Louis

Duchène, Denis

Dufresne, Tousst

Daigle, Alex.

Desjardins, Fr. Xr.

Desjardins, Flavien

Desjardins, Hilaire

Desloges, Antoine

Danis, Joseph

Demers, Eustache

Desjardins, Paul

Dagenais, Joseph

Desjardins, J. B

Denis, Antoine

Daoust, François

Dumouchelle, J. B to

Dumouchelle, Hercule

Dumouchelle, Camile

Daigneau, Christophe

Dubois, Louis

Decoigne, Ls. M.

Duverney, Joseph

Demers, Jacques

Duval, Joseph

Denis, Alex

Duval, Noël

Drouin, Hya.

Drouin, Alex.

Drolet, Alex.

Duchesneau, Noël

Dugas, Adolphe

Duvert, $\mathrm{Fr}^{\mathrm{s}}$ Chicou.

Desormeaux, J. Bte

Damour, Pierre

Dubeau, Jac. Cap ${ }^{\mathrm{n}}$

Donoghue, Henri

Desmarais, Joseph

$\begin{array}{lll}25 & \text { Commis } & \text { Montreal } \\ 51 & \text { Cultivateur } & \text { St Valentin } \\ 19 & \text { “ } & \text { Acadie } \\ 32 & \text { “ } & \text { St Jean } \\ 37 & \text { “ } & \text { Presentation } \\ 35 & \text { Marchand } & \text { Vaudreuil } \\ 34 & \text { Aubergiste } & \text { "“ } \\ 20 & \text { Cultivateur } & \text { Ste Scholastique } \\ 25 & \text { “ } & \text { St Eustache } \\ 40 & \text { “ } & \text { Ste Scholastique } \\ 18 & \text { “ } & \text { St Eustache } \\ 19 & \text { “ } & \text { Ste Scholastique } \\ 30 & \text { Menuisier } & \text { St Eustache } \\ 18 & \text { Cultivateur } & \text { Ste Scholastique } \\ 23 & \text { Voyageur } & \text { St Eustache } \\ 19 & \text { Cultivateur } & \text { “ } \\ 56 & \text { Marchand } & \text { St Benoit } \\ 22 & \text { “ } & \\ 18 & \text { Cultivateur } & \text { “" } \\ 30 & \text { Journalier } & \text { St Jn-Baptiste } \\ 39 & \text { Forgeron } & \text { Ste Anne Masc. } \\ 35 & \text { Notaire } & \text { Acadie } \\ 31 & \text { Menuisier } & \text { St Damase } \\ 30 & \text { “" } & \text { Ste Marie } \\ 36 & \text { Cultivateur } & \text { Acadie } \\ 23 & \text { Journalier } & \text { St Eustache } \\ 40 & \text { Cultivateur } & \text { Acadie } \\ 35 & \text { “ } & \text { Ste Scolastique } \\ 30 & \text { “ } & \text { St Benoit } \\ 22 & \text { Marchand } & \text { St Marc } \\ 40 & \text { “ } & \text { Ste Scholastique } \\ 20 & \text { Etud. medec. } & \text { Boucherville } \\ 49 & \text { Medecin } & \text { St Charles } \\ 25 & \text { Cultivateur } & \text { St Benoit } \\ 50 & \text { Marchand } & \text { Montréal } \\ 47 & \text { Cultivateur } & \text { St Eustache } \\ 54 & \text { Huissier } & \text { Montréal } \\ 18 & \text { Tonnelier } & \text { « } \\ & & \end{array}$

St Valenti

Acadie

t Jean

Vaudreuil

Ste Scholastiqu

Ste Scholastique

St Eustache

St Eustache

olastique

Marchand

Cultivateu

Journalier

Notaire

Cultivateur

Journalier
Fev. 28

oyez la

note (A)

"

"

$\begin{array}{llll}\text { Mars } & & & \\ \text { Fev. } & 28 & \text { “ } & \&(c)\end{array}$

Dec. 18

Fev. 28

Dec. 18

Janv. 24 “

Fer. 28

Mars 15

Voyez la

note (BB)

Janv. 27

Fev. 1

Dec. 15

Mev.

Mai

“. 10

“ 14

“ 21

\section{Mai}

Dec. 27

“ 10

Dec. 11 "

Voyez la

note $(\mathrm{H})$

Janv. 23

Mars 27 . 


\begin{tabular}{|c|c|c|c|c|c|c|c|c|c|c|c|}
\hline \multirow{12}{*}{$\begin{array}{l}\text { Dionne, Isidore } \\
\text { Dionne, Frs. } \\
\text { Dionne, Placide } \\
\text { Drolet, Jos Touss } \\
\text { Desvoyaux, Joseph } \\
\text { Delfosses, Alexis A. } \\
\text { Desrochers, Hubert } \\
\text { Demeule, Fr. } \\
\text { Dumouchelle, Jn. Bte } \\
\text { Deneau, Eustache } \\
\text { Ducharme, Ls. } \\
\text { Dewer, Ml. } \\
\text { Deshaîtres, Damase }\end{array}$} & \multirow{2}{*}{$\frac{\mathrm{AGE}}{24}$} & \multirow{2}{*}{$\begin{array}{c}\begin{array}{c}\text { PROFESSIONS } \\
\text { et autres } \\
\text { qualifications }\end{array} \\
\text { Cultivateur }\end{array}$} & DEMEURES & \multicolumn{2}{|c|}{$\begin{array}{l}\text { DATE } \\
\text { de leur } \\
\text { arrestation } \\
\text { ou } \\
\text { emprison- } \\
\text { nement }\end{array}$} & \multicolumn{2}{|c|}{$\begin{array}{c}\begin{array}{c}\text { Prisonniers } \\
\text { qui ont été } \\
\text { élargis }\end{array} \\
\begin{array}{c}\text { Date de la } \\
\text { sortie }\end{array}\end{array}$} & \multirow[t]{2}{*}{$\begin{array}{l}\text { sans } \\
\text { caution }\end{array}$} & \multirow[t]{2}{*}{$\begin{array}{l}\text { sous } \\
\text { caution }\end{array}$} & \multirow[t]{2}{*}{$\begin{array}{l}\text { Nombre de } \\
\text { jours de } \\
\text { détention, } \\
\text { au 1er } \\
\text { Juin } 1838\end{array}$} & \multirow[t]{2}{*}{$\begin{array}{c}\text { Indication } \\
\text { des } \\
\text { notes }\end{array}$} \\
\hline & & & St Césaire & “ & 30 & "6 & 28 & & & & \\
\hline & 22 & “6 & 6 & “ & “" & “ & “ & “" & & & \\
\hline & 30 & “ & “ & “ & “ & “ & “ & “ & & & \\
\hline & 51 & M.P.P. & St Marc & “" & 31 & & & & & & Voyez la \\
\hline & & $\underset{\text { Cultivateur }}{ }$ & $\begin{array}{l}\text { Ste Scholastique } \\
\text { St Hyacinthe }\end{array}$ & & $\begin{array}{l}1 \\
4\end{array}$ & $\begin{array}{l}\text { Fev. } \\
\text { Janv. }\end{array}$ & $\begin{array}{r}28 \\
6\end{array}$ & “" & & & $\begin{array}{l}\text { voyez la } \\
\text { note }(\mathrm{H})\end{array}$ \\
\hline & 20 & Sculpteur & Pte aux Trembles & “ & 26 & Fev. & 28 & “ & & & \\
\hline & 48 & Cultivateur J.P. & St Philipe & Dec. & 15 & & 46 & “ & & & \\
\hline & 32 & Cultivateur & Ste Scholastique & Fev. & 6 & & & & & & \\
\hline & 13 & & St Eustache & Dec. & 14 & Dec. & 18 & “ & & & \\
\hline & $\begin{array}{l}60 \\
45\end{array}$ & Forgeron & Ste "Marie & Fev & 5 & Avril & & “" & & & \\
\hline & $\begin{array}{l}43 \\
32\end{array}$ & Cuivateur & St Hermas & Avril & 28 & Avril & & & & & \\
\hline $\begin{array}{l}\text { Ethier, Jn Bte } \\
\text { Ethier, Louis }\end{array}$ & 57 & & St Valentin & Fev $^{r}$ & 15 & & & & & & \\
\hline Forlé, Antoine & 50 & Maçon & St Mathias & Nov $r$ & 30 & Fev. & 15 & “ & & & \\
\hline Foretier, Joseph & 50 & Journalier & 6 & 4 & “ & 4 & & “ & & & \\
\hline $\begin{array}{l}\text { Faneuf, P. Claude } \\
\text { Forbes dit McNaughton }\end{array}$ & 48 & Marchand & St Damase & “ & 18 & Janv. & 23 & $"$ & & & \\
\hline $\begin{array}{l}\text { Dan. } \\
\text { Fréchette Insenh }\end{array}$ & 35 & Cultivateur & St Eustache & $\operatorname{Dec}^{r}$ & 4 & Dec. & 5 & & “ & & \\
\hline $\begin{array}{l}\text { Fréchette, Joseph } \\
\text { Faneuf, Joseph }\end{array}$ & $\begin{array}{l}50 \\
40\end{array}$ & $\begin{array}{l}\text { Aubergiste } \\
\text { Journalier }\end{array}$ & St Jean & “ & $\begin{array}{r}9 \\
12\end{array}$ & & & “" & & & Voyez la \\
\hline Fournier, Alex. & 22 & Marchand & Ste Scholastique & “ & 14 & & & & & & note $(A)$ \\
\hline Falerdau, Chs. & 20 & Cultivateur & & “ & $" 6$ & Dec. & 18 & “ & & & \\
\hline Fontaine, $\mathrm{D}$. & 35 & Journalier & St Jérôme & “ & “ & 4 & 14 & “" & & & \\
\hline Franchère, Timothé & 47 & Marchand & St Mathias & Janv. & 5 & & & & & & Voyez la \\
\hline Foisy, Isaïe & 43 & Forgeron & St Eustache & 6 & 6 & & & & & & note $(\mathrm{H})$ \\
\hline Fréjou, Michel & 23 & Cultivat $^{t}$ & St Césaire & “ & 10 & Avril & 11 & “ & & & \\
\hline $\begin{array}{l}\text { Giguères, André } \\
\text { Gouin, Chs. }\end{array}$ & $\begin{array}{l}30 \\
50\end{array}$ & $\begin{array}{l}\text { Forgeron } \\
\text { Aubergiste }\end{array}$ & $\begin{array}{l}\text { Montréal } \\
\text { Sorel }\end{array}$ & Dec. & $\begin{array}{l}1 \\
4\end{array}$ & $\begin{array}{l}\text { Dec. } \\
\text { Mai }\end{array}$ & $\begin{array}{r}15 \\
9\end{array}$ & “" & & & \\
\hline
\end{tabular}


Gareau, Alarie

Gauvin, Henri

Godhue, Touss.H.

Gratton, W.

Grignon, Frs. $X^{\mathbf{r}}$

Groulx, Ant.

Guindon, $\mathbf{M g}^{\mathrm{re}}$

Guérin, Bertrand

Gareau, Am ${ }^{l e}$

Gareau, Et ${ }^{\text {ne }}$

Guilard, Arsène

Gosselin, Isidore

Guertin, Frs. $\mathbf{X}^{\mathrm{r}}$

Girouard, Jos.

Gigon, Calixte

Guérin, Frs.

Gauthier dit St-Germain

$$
\text { Joseph }
$$

Glouttiné, Olivier

Gervais, Jos

Guillaume, Jean

Gérardin, Zéphirin

Gaudin de la Poterie

$$
\text { Barth. }
$$

Gagnon, Médard

Gerard, Geo.

Garriépy, Jos.

Grenier, Pierre

Gaudet, $\mathbf{M}^{1}$

Gibson, Wm.

Hebert, Frs.

Hebert, Ambso

Hubert, Richard

Hubert, Frs.-Xr

Hennesey, Denis

Hebert, Luc

\begin{tabular}{|c|c|c|}
\hline 20 & Marchand & St Césaire \\
\hline 23 & Médecin & Montréal \\
\hline 44 & Cultiv. Majr I & M.St-Césaire \\
\hline 27 & Cultivat. & Ste Scholastiq \\
\hline 46 & " & St Eustache \\
\hline 58 & $"$ & St Eustache \\
\hline 28 & " & " \\
\hline 43 & M.P.P. & St Benoit \\
\hline 63 & Cultivateur & St Eustache \\
\hline 18 & “" & St Jérôme \\
\hline 4 & “" & \\
\hline 22 & Macan & St Eustache \\
\hline 18 & Maçon & \\
\hline 42 & Charpentier & St Césaire \\
\hline 50 & Cultivateur & St Eustache \\
\hline 29 & Marchand & St Césaire \\
\hline 61 & Cultivateur & St Eustache \\
\hline 48 & Marchand & St Edouard \\
\hline 17 & Journalier & Contrecœur \\
\hline 19 & Cultiv. & \\
\hline 50 & Huissier & St Laurent \\
\hline 21 & Cultivateur & Acadie \\
\hline 60 & “" & Varennes \\
\hline 15 & " & St Valentin \\
\hline 20 & Imprim $^{\mathrm{r}}$ & Montréal \\
\hline 50 & Marchand & St George \\
\hline $\begin{array}{l}32 \\
30\end{array}$ & Cultivateur & Montreal \\
\hline & & \\
\hline 19 & Cultivateur & Verchères \\
\hline 25 & Avocat & Montréal \\
\hline 18 & Etud' en droit & St Denis \\
\hline 45 & Cultivateur & St Edouard \\
\hline 34 & Journalier & St Jean \\
\hline 40 & Cultivateur & St Cyprien \\
\hline
\end{tabular}

Hebert, Jos
(H)

Voyez la note $(\mathrm{H})$

“ 12 Fev.

Fev $^{r}$

Fevr

Mars 10

Mai 12

Mars 27

Dec 14 “.

“ 14

Nov. 30

$\operatorname{Dec}^{\mathrm{r}} 12$

Janv $^{\mathrm{r}} 5$

“ 5

Dec. 17

Janv. 25
" 29 "

Voyez la

note (A) 


\begin{tabular}{|c|c|c|c|c|c|c|c|c|c|c|c|}
\hline \multirow[b]{2}{*}{$\begin{array}{l}\text { Hebert, J. Bte } \\
\text { Huneau, J.B. } \\
\text { Huberdeau, Ant. }\end{array}$} & \multirow{2}{*}{$\begin{array}{l}\text { AGE } \\
\begin{array}{l}57 \\
45\end{array}\end{array}$} & \multirow{2}{*}{$\begin{array}{l}\begin{array}{c}\text { PROFESSIONS } \\
\text { et autres } \\
\text { qualifications }\end{array} \\
\begin{array}{l}\text { M.P.P. } \\
\text { Marchand } \\
\text { Cultivateur }\end{array}\end{array}$} & \multirow{2}{*}{$\begin{array}{l}\text { DEMEURES } \\
\text { Nicolet } \\
\text { St Lin } \\
\text { St Jérôme }\end{array}$} & \multicolumn{2}{|c|}{$\begin{array}{c}\text { DATE } \\
\text { de leur } \\
\text { arrestation } \\
\text { ou } \\
\text { emprison- } \\
\text { nement }\end{array}$} & \multicolumn{2}{|c|}{$\begin{array}{c}\text { Prisonniers } \\
\text { qui ont étó } \\
\text { ćlargis }\end{array}$} & \multirow{2}{*}{$\begin{array}{c}\begin{array}{c}\text { sans } \\
\text { caution }\end{array} \\
\text { " } \\
\text { " }\end{array}$} & \multirow{2}{*}{$\begin{array}{c}\text { sous } \\
\text { caution }\end{array}$} & \multirow[t]{2}{*}{$\begin{array}{c}\text { Nombre de } \\
\text { jours de } \\
\text { détention, } \\
\text { au 1er } \\
\text { Juin } 1838\end{array}$} & \multirow[t]{2}{*}{$\begin{array}{c}\text { Indication } \\
\text { des } \\
\text { notes }\end{array}$} \\
\hline & & & & $\begin{array}{l}\text { Fev. } \\
\text { Decr }\end{array}$ & $\begin{array}{r}4 \\
15 \\
17\end{array}$ & $\begin{array}{l}\text { Fev. } \\
\text { Dec. }\end{array}$ & $\begin{array}{l}27 \\
19 \\
18\end{array}$ & & & & \\
\hline $\begin{array}{l}\text { Joli, Joseph } \\
\text { Jabot, Jean } \\
\text { Jobin, André } \\
\text { Jalbert, Frs. }\end{array}$ & $\begin{array}{l}26 \\
17 \\
51 \\
64 \\
52\end{array}$ & $\begin{array}{l}\text { Journalier } \\
\text { M.P.P. } \\
\text { Cultivateur } \\
\text { Capne } \\
\text { Medecin }\end{array}$ & $\begin{array}{l}\text { Ste Scholastique } \\
\text { St Jean } \\
\text { Ste Geneviève } \\
\text { St Denis } \\
\text { Patton }\end{array}$ & $\begin{array}{l}\text { Decr } \\
\text { Janv. } \\
\text { Mai } \\
\text { Decr } \\
\text { Avril }\end{array}$ & $\begin{array}{r}14 \\
25 \\
3\end{array}$ & $\operatorname{Dec}^{r}$ & 18 & “ & & & $\begin{array}{l}\text { Voyez la } \\
\text { note (A) }\end{array}$ \\
\hline Kimber, Timothé & 41 & Médecin & Chambly & $\operatorname{Dec}^{r}$ & 6 & Janv. & 11 & ، & & & $\begin{array}{l}\text { Voyez la } \\
\text { note (A) } \\
\text { " (H) }\end{array}$ \\
\hline $\begin{array}{l}\text { Leblanc, Ch.A. } \\
\text { Lyonnais, Jn Frs Bossu } \\
\text { Lamothe, Narcisse } \\
\text { Lacroix, André } \\
\text { Lavallé, Domque } \\
\text { L'Heureux, Pre } \\
\text { Legros, Pre } \\
\text { Leduc, J.Bte } \\
\text { Lussier, Olivier } \\
\text { Lussier, Pre } \\
\text { Lemire, Frs. } \\
\text { Lachapelle, Touss. } \\
\text { ou Augn } \\
\text { Lafontaine, J.B. } \\
\text { Landry dit Grandis, Jos. } \\
\text { Lecuier, David X }\end{array}$ & $\begin{array}{l}21 \\
46 \\
18 \\
30 \\
40 \\
47 \\
20 \\
26 \\
23 \\
25 \\
25\end{array}$ & $\begin{array}{l}\text { Etud' en droit } \\
\text { Medecin } \\
\text { Marchand } \\
\text { Medecin } \\
\text { Cultivateur } \\
\text { “" } \\
\text { “" } \\
\text { “" } \\
\text { Marchand }\end{array}$ & $\begin{array}{l}\text { Montréal } \\
\text { St Athanase } \\
\text { Prairie } \\
\text { Montréal } \\
\text { St Athanase } \\
\text { St Hilaire } \\
\text { St Hyacinthe } \\
\text { St Charles } \\
\text { " } \\
\text { St Jean Baptiste } \\
\text { St Charles }\end{array}$ & $\begin{array}{c}\text { Nov. } \\
\text { “" } \\
\text { “ } \\
\text { “" } \\
\text { “ } \\
\text { " } \\
\text { “ } \\
\text { " }\end{array}$ & $\begin{array}{l}16 \\
17 \\
24 \\
\text { " } \\
28 \\
30 \\
30 \\
\text { ". } \\
\text { “ } \\
26\end{array}$ & $\begin{array}{l}\text { Mars } \\
\text { Mars } \\
\text { Decr }^{r}\end{array}$ & $\begin{array}{l}18 \\
4 \\
6\end{array}$ & $\begin{array}{l}\text { ". } \\
. . \\
. \\
" . \\
\text { ". } \\
\text { " }\end{array}$ & & & $\begin{array}{l}\text { Voyez la } \\
\text { note (BB) } \\
\text { Voyez la } \\
\text { note (a) }\end{array}$ \\
\hline
\end{tabular}




Languedoc, J.Bte
Lafond, Frs.
Languedoc, pre
Lauzon, Aug
Lafleur, Petit
Leclerc, André ou Ant.
Laplante, Benj.
Lefebvre, Pre
Ladouceur, Marc
Leroux, Charles
Labelle, Frs.
Landry, J.Bte
Laurin, Philibert
Laurin, Vincent
Lespagnol, Anselme
Larose, Chs.
Laurin, Paul
Ladouceur, Fr.
Lacoste, Boromée
Lapierre, J.Bte
Laroque, FrsXr.
Lafantaisie, Jean
Laplante, Ant.
Laurent dit Lortie, Augn
Lapierre, Ambse
Langlois, Tousst
Laporte, Jos.
Lorimier, Gédéon
Lemoine, Chs.
Lapierre, Mazurette Jos
Lorimier, Geo.
Lentier, Olivier
Labre, Emery
Lacombe, J. Bte
Labrie, Auguste

$\begin{array}{lc}26 & \text { Journalier } \\ 45 & \text { Cultivateur } \\ 36 & \text {. }\end{array}$

St Damase

“ 12

Journalier Cultivateur

Journalier

Tonnellier

Cultivateur

Ile Bizare

St Eustache

Ste Scholastique

St Jérôme

Ste Scholastique

St Jérôme

Ste Scholastique

St Eustache

Cultivat $^{r}$

St Jérôme

$$
\text { " }
$$

St Eustache

"

"

Journalier

Ste Scholastique

Etud' en méd. Montréal

Orfèvre

Cultivat ${ }^{r}$

Marchand

Cultivat ${ }^{r}$

Marchand

St Jean B

$\operatorname{Dec}^{x}$

note (a)

Cultivat

Sault St Louis

Montréal

Coteau du Lac

Ste Scholastique

St Jérôme

" " "

“.

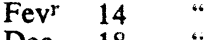

Dec. 18

" 6 ".

"

Fev ${ }^{r}$

$\begin{array}{ccc}\operatorname{Dec} & 18 & . .\end{array}$

Dec. 18 *

$\begin{array}{lll}" & 4 & . \\ " & 4 & .\end{array}$

Dec. 18

Fev.

•.

Mai 


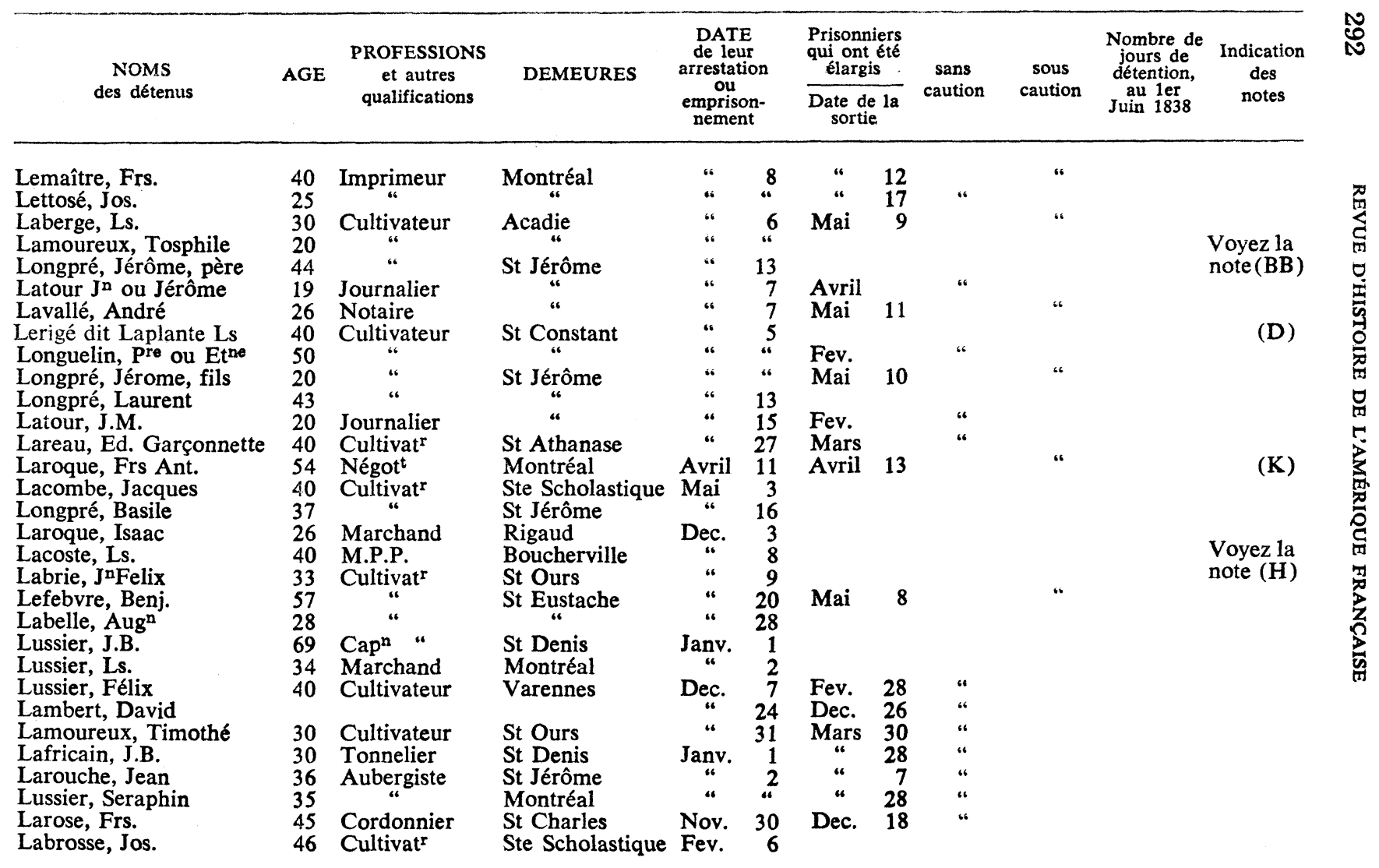


Monjeau, Ls., père

Monjeau, Ls., fils

Menard, Jos.

Martin, Chs.

Maugé, Ls.

Mercile, Touss ${ }^{t}$

Marchesseau, Siméon

Marchesseau, Thos.

Masson, Luc Hya.

Masson, Damien

Mallet, Frs.

Mallet, Jos.

Monnet, Aug ${ }^{n}$

Marineau, Aug

Maisonneuve, Flavien

Maisonneuve, Frs.

Major, Edouard

Malboeuf, Joseph

Mainville, Hya.

Major, Martial

Masson, $\mathbf{M}^{\mathbf{l}}$

Meloche, Frs.

Marié, Léon

Martel, Am.

Maugé, Hyp.

Marié, pre

Mongeon, Chs.

Marchand, Ls.

Mallet, Vital

Montplaisir, Wm.

Monet, Julien

Monet, Pre

McMahon, Robert

Murray, Patrick

Marchesseau, Moïse

Mondore, pre

\begin{tabular}{|c|c|c|}
\hline $\begin{array}{l}55 \\
36 \\
23 \\
25 \\
50 \\
45 \\
31\end{array}$ & $\begin{array}{l}\text { Marchand } \\
\text { Forgeron } \\
\text { Huissier }\end{array}$ & $\begin{array}{l}\text { St Ours } \\
\text { Montréal } \\
\text { St Charles }\end{array}$ \\
\hline $\begin{array}{l}36 \\
27 \\
21 \\
58 \\
21 \\
28 \\
21 \\
20 \\
50 \\
27 \\
54 \\
34 \\
20 \\
33 \\
18 \\
28 \\
40 \\
48 \\
18 \\
37\end{array}$ & $\begin{array}{l}\text { Forgeron } \\
\text { Medecin } \\
\text { Marchand } \\
\text { Cultivat" } \\
\text { "“ } \\
\text { ". } \\
\text { “" } \\
\text { Marchand } \\
\text { Voyageur } \\
\text { Cultivateur } \\
\text { Charpentier } \\
\text { Menuisier } \\
\text { Cultivat } \\
\text { Meunier } \\
\text { Menuisier } \\
\text { Marchand } \\
\text { “" }\end{array}$ & $\begin{array}{l}\text { St Hyacinthe } \\
\text { St Benoit } \\
\text { St Benoit } \\
\text { St Eustache } \\
\text { “" } \\
\text { Ste Scholastique } \\
\text { St Eustache } \\
\text { “" } \\
\text { Ste Scholastique } \\
\text { St Eustache } \\
\text { St Jàrome } \\
\text { St Eustache } \\
\text { St Eustache } \\
\text { Ste Scholastique } \\
\text { St Eustache } \\
\text { Ste Scholastique } \\
\text { St Ours } \\
\text { St Eustache } \\
\text { St Athanase }\end{array}$ \\
\hline $\begin{array}{l}38 \\
40 \\
44 \\
19 \\
17 \\
21 \\
54 \\
40 \\
20\end{array}$ & $\begin{array}{l}\text { Cultivat } \\
\text { "“ } \\
\text { "“ } \\
\text { Voyageur } \\
\text { Cultivat } \\
\text { Forgeron } \\
\text { Pottier }\end{array}$ & $\begin{array}{l}\text { St Mathias } \\
\text { Pte Claire } \\
\text { St Césaire } \\
\text { St Edouard } \\
\text { “ } \\
\text { Montréal } \\
\text { Ste Marie } \\
\text { Acadie } \\
\text { St Denis }\end{array}$ \\
\hline
\end{tabular}

Voyez la

note (A)

Voyez la

note (bb)

(f)

马्8

Voyez la

note $(\mathrm{h})$

Voyez la

note $(h)$ 


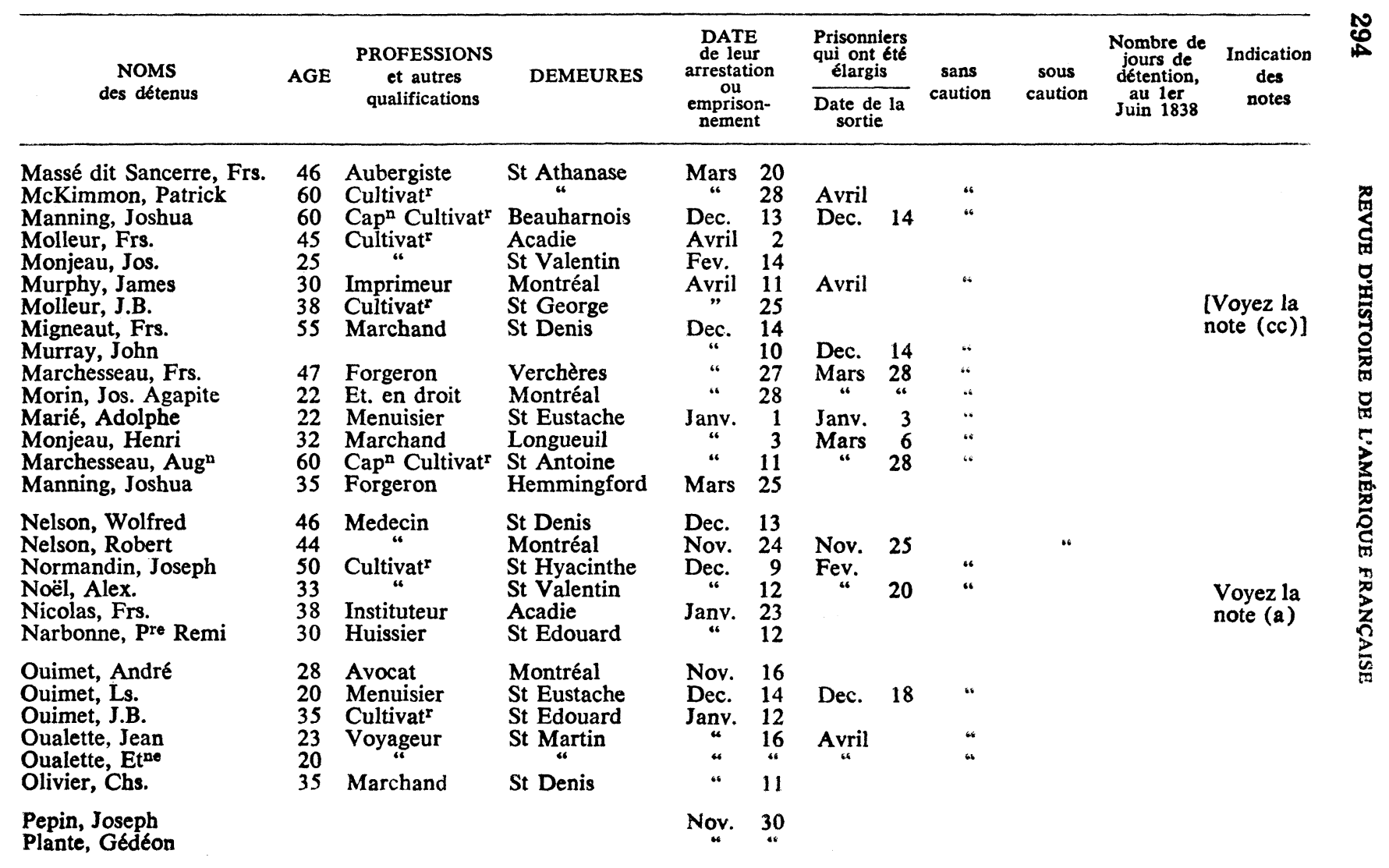


Peltier, Touss ${ }^{t}$

Peloquin, Denis

Pinsoneau, Gédéon

Pinsoneau, Joseph

Parent, Louis

Provost, Narcisse

Pinsoneau, Alex ${ }^{\mathrm{re}}$

Parent, Celestin

Poirier, Benj.

Prevost, Paul

Poisson, Barthelemi

Papineau, André Aug ${ }^{n}$

Proulx, J.B.

Papineau, Louis

Pilon, Frs

Petit dit Lalumière, Jos.

Prevost, Théophile

Papineau, André Benj ${ }^{n}$

Poulin, Cap ne Louis

Paradis, Amb.

Perrault, L. Calixte

Perrault, Joseph

Peltier, Louis

Renault, Hubert

Reignier, Julien

Roy, David

Reignier, Benonie

Reignier, Louis

Richard, Alexis

Roberge, Pierre

Roberge, Olivier

Robert, Thos

Rochon, Olivier

Robillard, Joseph

Robillard, J. Bte

Rochon, Isidore

Rocbrune, Jn Marie

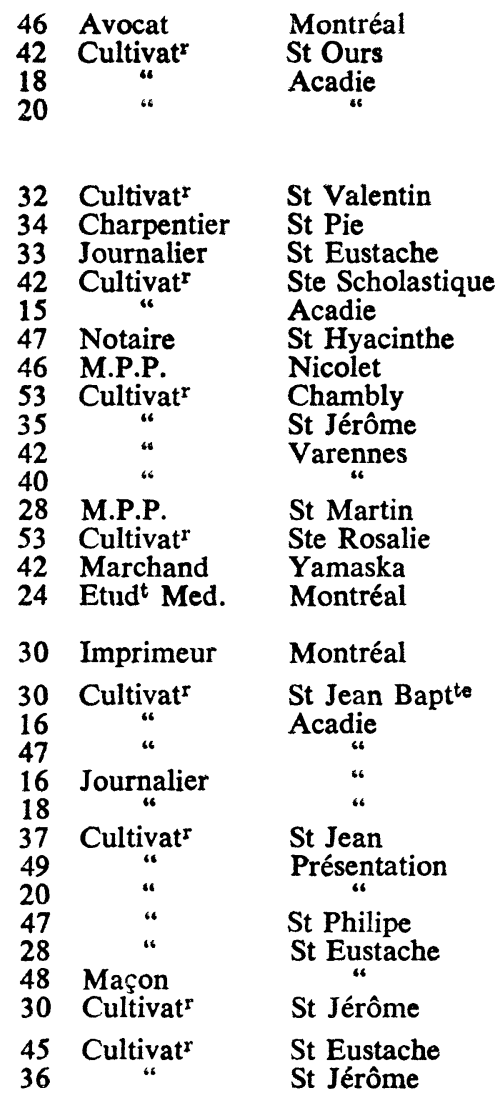

\begin{tabular}{|c|c|c|c|}
\hline Dec. & $\begin{array}{l}1 \\
4\end{array}$ & Fev. & \\
\hline 4 & 9 & & \\
\hline " & 4 & & \\
\hline 6 & " & & \\
\hline$"$ & 12 & & \\
\hline$"$ & 15 & Fev. & \\
\hline " & 17 & Mars & \\
\hline " & 14 & Fev. & \\
\hline Janv. & 25 & & \\
\hline & $\begin{array}{l}2 \\
4\end{array}$ & Fev. & 27 \\
\hline " & 5 & Avril & \\
\hline “ & 8 & Mai & 15 \\
\hline$“$ & 12 & Fev. & \\
\hline "6" & 20 & & \\
\hline $\begin{array}{l}\text { Dec. } \\
\text { Jany }\end{array}$ & & & \\
\hline $\begin{array}{l}\text { Janv. } \\
\text { Mars }\end{array}$ & 28 & Mai & 7 \\
\hline Dec. & 13 & Dec. & 14 \\
\hline Janv. & 23 & Mars & 28 \\
\hline Nov. & $\begin{array}{r}30 \\
9\end{array}$ & Dec. & 18 \\
\hline Dec. & $\begin{array}{r}9 \\
12\end{array}$ & Fev. & 20 \\
\hline “ & $"$ & Dec. & 18 \\
\hline " & "6 & & \\
\hline " & “ & & \\
\hline “" & 15 & Mars & \\
\hline "“ & “" & $" 4$ & \\
\hline " & 16 & Fev. & 28 \\
\hline Dec. & 26 & & \\
\hline & 17 & Dec. & 18 \\
\hline$"$ & $\because$ & Janv. & \\
\hline “" & 14 & Dec. & 18 \\
\hline
\end{tabular}

Voyez la

note (a)

Voyez la note (a) 


\begin{tabular}{|c|c|c|c|c|c|c|c|c|c|c|c|}
\hline $\begin{array}{c}\text { NOMS } \\
\text { des détenus }\end{array}$ & AGE & $\begin{array}{c}\text { PROFESSIONS } \\
\text { et autres } \\
\text { qualifications }\end{array}$ & DEMEURES & \multicolumn{2}{|c|}{$\begin{array}{l}\text { DATE } \\
\text { de leur } \\
\text { arrestation } \\
\text { ou } \\
\text { emprison- } \\
\text { nement }\end{array}$} & \multicolumn{2}{|c|}{$\begin{array}{c}\begin{array}{c}\text { Prisonniers } \\
\text { qui ont été } \\
\text { élargis }\end{array} \\
\begin{array}{c}\text { Date de la } \\
\text { sortie }\end{array}\end{array}$} & $\begin{array}{l}\text { sans } \\
\text { caution }\end{array}$ & $\begin{array}{l}\text { sous } \\
\text { caution }\end{array}$ & \multirow[t]{2}{*}{$\begin{array}{c}\text { Nombre de } \\
\text { jours de } \\
\text { détention, } \\
\text { au 1er } \\
\text { Juin } 1838\end{array}$} & $\begin{array}{c}\text { Indication } \\
\text { des } \\
\text { notes }\end{array}$ \\
\hline Rochon, Ant ne & 47 & “ & St Eustache & “ & 17 & Fev. & & $“$ & & & \multirow{7}{*}{$\begin{array}{c}\text { note }(\mathrm{bb}) \\
(\mathrm{g})\end{array}$} \\
\hline Renault, Frs. & 34 & “ & “" & $“$ & 20 & Mars & & $“$ & & & \\
\hline Robillard, Ulric & 22 & Maçon & “ & “ & $"$ & Janv. & 22 & $"$ & & & \\
\hline Roy, Stanislas & 32 & Cultivat ${ }^{r}$ & Kakouna & $"$ & $“$ & & & & & & \\
\hline Roy, Modeste & 18 & Forgeron & St Denis & $"$ & 15 & & & & & & \\
\hline Robitaille, Ls.Adolphe & 36 & Notaire & Varennes & " & 20 & Fev. & & $"$ & & & \\
\hline Rousse, Joseph & 46 & Cultivat ${ }^{x}$ & Pte Claire & Janv. & 8 & Janv. & & “. & & Voyez la & \\
\hline $\begin{array}{l}\text { Ranger, Frs. } \\
\text { Roy, Théophile }\end{array}$ & $\begin{array}{l}27 \\
30\end{array}$ & $\begin{array}{l}\text { Marchand } \\
\text { Cultivat }\end{array}$ & $\begin{array}{l}\text { Acadie } \\
\text { St George }\end{array}$ & Dec. & $\begin{array}{l}20 \\
25\end{array}$ & & & $" “$ & & & (g) \\
\hline Robert, Aug ${ }^{n}$ & 45 & “ & St Philipe & Janv. & 29 & & & “ & & & \\
\hline Robillard, Jos., fils & 24 & Maçon & St Eustache & Dec. & 14 & & & & & & \\
\hline $\begin{array}{l}\text { Rousseau, Jos. O. } \\
\text { Richer, J. B }\end{array}$ & $\begin{array}{l}31 \\
30\end{array}$ & $\begin{array}{l}\text { Medecin } \\
\text { Marchand }\end{array}$ & $\begin{array}{l}\text { Nicolet } \\
\text { St Benoit }\end{array}$ & Fev. & $\begin{array}{r}4 \\
10\end{array}$ & Fev. & 27 & “" & & & \\
\hline Richer, Frs. & 20 & Cultivat ${ }^{x}$ & St Denis & Fev. & 17 & Fev. & & “ & & & \\
\hline Rolland, Nicolas & 39 & Cordonnier & St Antoine & Dec. & 6 & & & & & & \\
\hline $\begin{array}{l}\text { Simard, Am }{ }^{\mathrm{b}} \\
\text { Sabourin, Narcisse }\end{array}$ & 33 & Medecin & Montréal & Nov. & $\begin{array}{l}16 \\
30\end{array}$ & & & & & & \\
\hline $\begin{array}{l}\text { Spenard, Gilbert } \\
\text { Soucy, Ant }{ }^{\text {ne }}\end{array}$ & 22 & Menuisier & St Eustache & Dec. & $\begin{array}{r}14 \\
9\end{array}$ & Dec. & 18 & $"$ & & & \\
\hline Sicot, Tousst. & 26 & Etud' en droit & St Hyacinthe & “ & 4 & Fev. & & $"$ & & & \\
\hline Surprenant, Jacques & 30 & $\begin{array}{l}\text { Aubergiste } \\
\text { Cultivat }^{5}\end{array}$ & Acadie & “ & 15 & & & & & & \\
\hline Spenard, Flavien & 54 & Menuisier & St Eustache & $“$ & 14 & Dec. & 18 & . & & & \\
\hline Scott, Wm H. & 36 & M.P.P. & " c. & " & 17 & & & & & & Voyez la \\
\hline $\begin{array}{l}\text { Soupras, Eust. } \\
\text { Stenback, Henry }\end{array}$ & 49 & Marchand & St Mathias & Janv. & 5 & Fev. & 27 & " & & & note $(h)$ \\
\hline Sanche, Aug ${ }^{\mathrm{n}}$ & 44 & Cultivat $^{\mathbf{r}}$ & Ste Scholastique & $“$ & 6 & & & & & & \\
\hline Seguin, Frs. & 21 & Boulanger & St Athanase & “ & 10 & Janv. & & $"$ & & & \\
\hline Sené, Marcel & 38 & Cultivat ${ }^{r}$ & St Césaire & $"$ & 6 & Avril & 11 & $" 6$ & & & \\
\hline
\end{tabular}




Surprenant, Frs., père
Senecal, Benj.
Scott, Neil S.
Senecal, J. Bte
Sanche, Anselme
Sicot, J.Bte
Tavernier, Frs.
Taillé, Jos.
Thompson, John
Tremblé, Julien
Tremblé, Louis
Trudeau, Laurent
Tetreau, J. Bte
Tetreau, Benj.
Tougas, M1
Tougas, Frs.
Tassé, Joseph
Thibault, Laurent
Trotier, Moïse
Tremblé, Etne
Tellier, Jos
Tremblé, J.Bte
Tougas, Jos
Touron, Désiré
Tessier, Paschal
Viger, Ls. M ${ }^{1}$
Vincent, M
Vaudry, Jacob
Vidal, Chs.
Vanchestaing, J. Bte
Valiquet, Anselme ou
Isidore
Valiquet, Ls.
Vilaire, Edouard
Vallé, J.Bte
Vadenais, Jos
Viger, Paschal

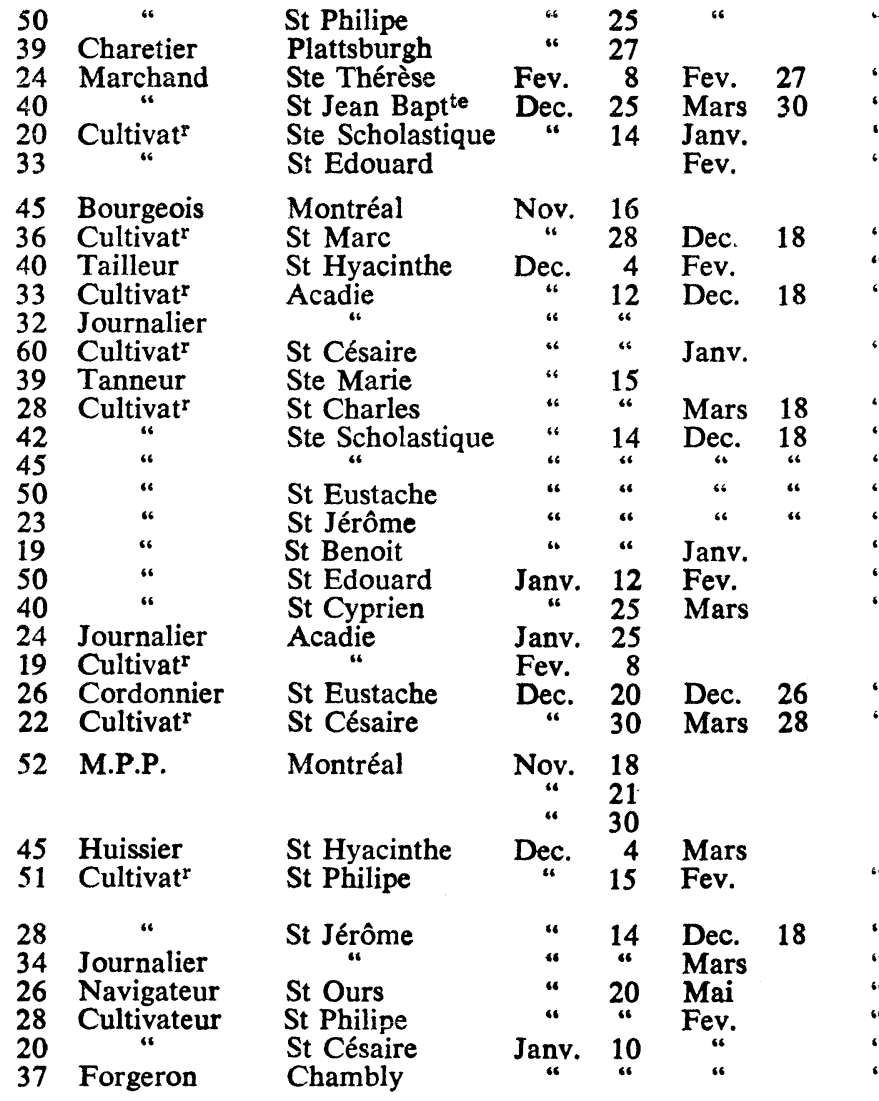

Voyez la note (a) 


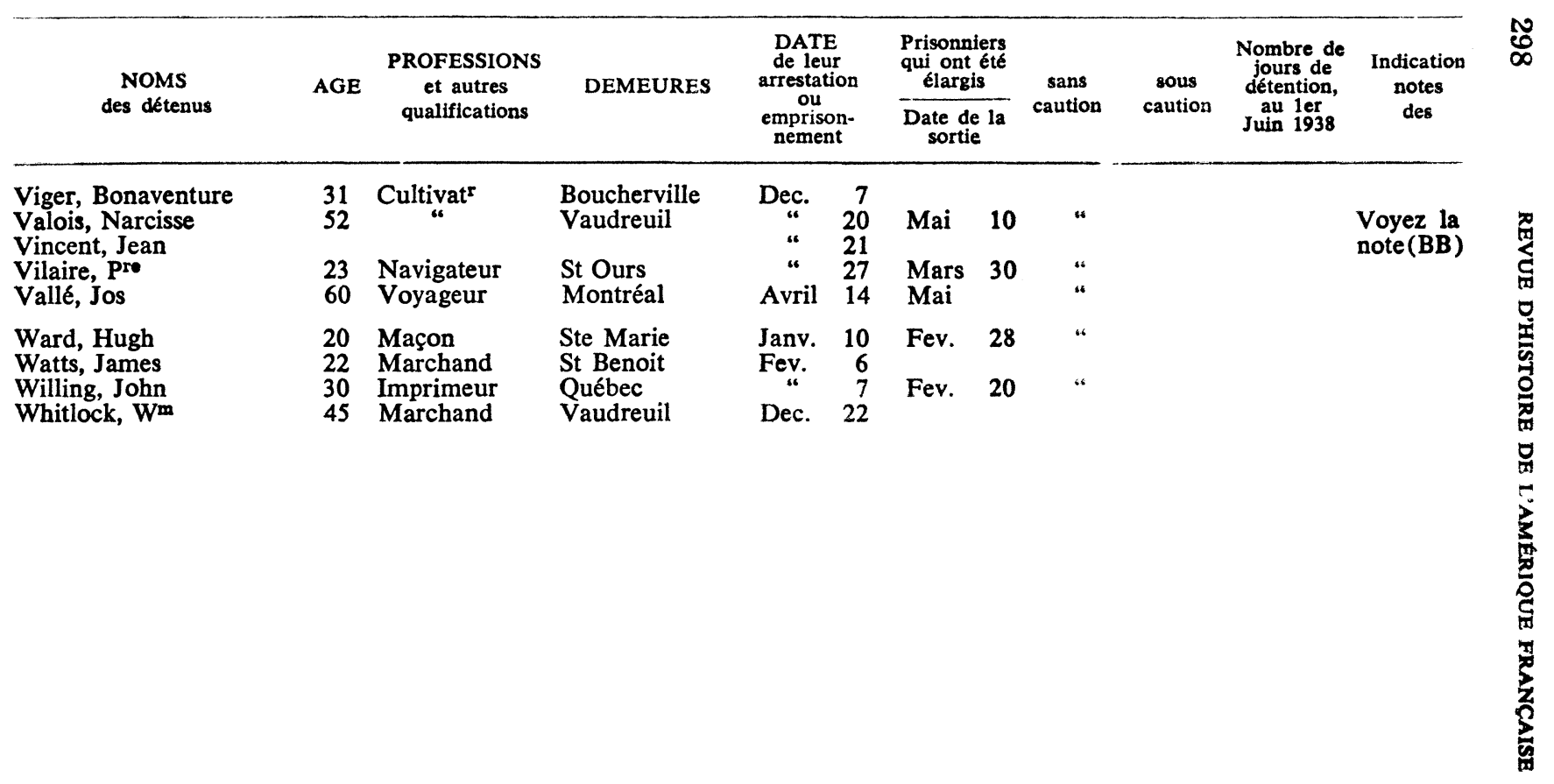




\section{RÉCAPITULATION}

\section{Classification}

Prêtres

Membres du Parlement Provincial

Avocats, outre 2 qui sont M.P.P.

Notaires, outre 4 " " "

Médecins outre 1 “" "

Arpenteurs

Marchands outre 3 " " "

Instituteurs

Journalistes et Imprimeurs de papiers-nouvelles

Etudiants en droit et en médecine

Bourgeois

Cultivateurs outre 3 qui sont M.P.P.

Artisans

Aubergistes

Voyageurs, journaliers \&c.

Huissiers

Dont la qualification n'est pas connue

Total des Patriotes incarcérés à Montréal

\section{ÂGES}

Au dessous de 20 ans

De 20 à 30 ans

De 30 à 40 “"

De 40 à 50 "

110

De 50 à 60 "

50

De 60 et au dessus

Dont l'âge n'est pas connu

Dans et hors la Prison au $1^{\text {er }}$ juin 1838.

Les Patriotes qui sont actuellement en prison y sont repartis ainsi qu'il suit:

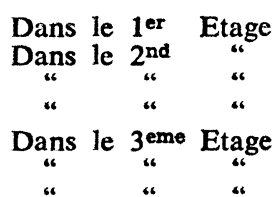

Dans l'Attique
Cachots de l'Aile N.O.

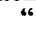

“

“ S.O.

Chambres " "

N.O.

Appartemens du centre (destiné à servir de Chapelle).

$1^{\text {er }}$ juin 1838.

Total des détenus politiques

Elargis avec cautionnement de $£ 300$ à $£ 4000$

Elargis, on ne sait pas le jour, ni si c'est avec ou sans cautionnement

Total pareil. 
Temps de détention

des Patriotes qui ont été incarcérés à Montréal

$\mathrm{Au}$ dessous d'un mois 105 Dont restant en prison au 1 juin

1 mois \& au-dessus

2 " " 6 "

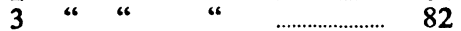

4 c 4 c

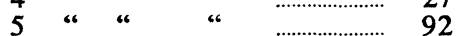

6 c " c 612

Dont le temps de détention est inconnu

\begin{tabular}{|c|c|c|c|c|}
\hline Dont & $\underset{\text { restant en }}{\text { en }}$ & prison au & 1 juin & …………......... \\
\hline ،6 & “ & s & 66 & . \\
\hline ،6 & “ & “ & “ & \\
\hline a6 & “ & “ & " & \\
\hline “ & “ & “ & “ & 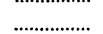 \\
\hline "6 & 4 & “ & $\omega$ & 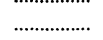 \\
\hline "6 & “" & “ & “ & ............. \\
\hline
\end{tabular}

Total pareil $\overline{495}$ Total des prison"s resiant au $\mathcal{L}^{\text {er Juin }} 1838 \overline{145}$

Acadie 1'

de

\section{RÉSIDENCE}

Beauharnois

Boucherville

Chambly

Contrecœur

Coteau-du-Lac

Dunham

Godmanchester

Hemmingford

Ile Bizare

Kakouna

Longueuil

Montréal

Milton

Nicolet

Présentation-la'

Pointe-aux-trembles

Pointe-claire

Patton

Prairie la'

Plattsburgh

Québec

Rigaud

St Laurent

Ste Marie

St Marc

St Martin

St Mathias

St Ours

St Pie

St Philipe

Ste Scholastique

\begin{tabular}{|c|c|c|}
\hline & & \\
\hline 27 & Sorel & 2 \\
\hline 1 & Stanbridge & 1 \\
\hline 3 & St Athanase & 10 \\
\hline 4 & Ste Anne de Mascouche & 1 \\
\hline 2 & St Antoine & 2 \\
\hline 1 & St Benoît & 16 \\
\hline 1 & St Charles & 14 \\
\hline 1 & St Cesaire & 18 \\
\hline 1 & St Cyprien & 3 \\
\hline 5 & St Constant & 2 \\
\hline 1 & St Denis & 14 \\
\hline 1 & St Damase & 6 \\
\hline 39 & St Eustache & 77 \\
\hline 4 & St Edouard & 15 \\
\hline 3 & St George & 3 \\
\hline 7 & Ste Geneviève & 1 \\
\hline 1 & St Hilaire & 2 \\
\hline 4 & St Hyacinthe & 9 \\
\hline 1 & St Hermas & 1 \\
\hline 1 & St Jean Baptiste & 6 \\
\hline 1 & St Jérôme & 30 \\
\hline 2 & St Jean & 8 \\
\hline 1 & St Lin & 2 \\
\hline 1 & Sault St Louis & 1 \\
\hline 6 & Ste Rosalie & 2 \\
\hline 4 & Ste Thérèse & $\overline{1}$ \\
\hline 3 & St Valentin & 11 \\
\hline 3 & Varennes & 5 \\
\hline 11 & Vaudreuil & 5 \\
\hline 1 & Verchères & 3 \\
\hline 7 & Yamaska & 3 \\
\hline 41 & Dont la demeure est inconnue & 32 \\
\hline 189 & ci-contre & 189 \\
\hline & Total pareil. & 495 \\
\hline
\end{tabular}




\section{LISTE DES PATRIOTES}

prévenus de pratiques séditieuses, \&c. emprisonnées (sic) à Québec en novembre 1837, et relachées (sic) ensuite, sous cautionnement pour comparaître, \&c. après plusieurs jours d'incarcération.

\begin{tabular}{|c|c|c|c|c|}
\hline Augustin-Norbert & Morin & 33 ans & M.P.P. de & Québec \\
\hline Eugêne & Trudeau & $41 “$ & Marchand & “ \\
\hline Joseph & Legaré (fils) & 42 & Peintre & “ \\
\hline Barthelemy & Lachance & 49 & Marchand & “ \\
\hline Pierre & Chasseur & 50 & Zoologiste & “ \\
\hline Robert & Layfield & 45 & Marchand & Leeds \\
\hline J.B. & Ryan & 22 & Commis & Québec \\
\hline John & Ryan & 50 & Agent & " \\
\hline
\end{tabular}

\section{LISTE DES PATRIOTES}

prévenus de haute trahison, \&c. incarcérés dans la prison des Trois-Rivières, et élargis ensuite avec ou sans cautionnement oûtre A. Augustin Papineau. Ecuier qui, ayant été emprisonné aux Trois-Rivières, et ensuite admis à caution, a été aussitôt après conduit prisonnier à Montréal, et est en conséquence compris dans le Grand Tableau qui précède. M.M. Proulx \& Hébert \& le Dr J.O. Rousseau, tous trois du district des Trois-Rivières ont été amenés dans les prisons de Montréal et sont compris dans le même Tableau.

Jean-Baptiste Courchêne, 40 ans, cultivateur de St François, arrêté au commencernent de Janvier et demeuré 17 jours en prison.

Pierre Cartier, $\quad 40$ Cultivateur St François, arrêté au commencePierre Cartier, ment de Janvier, est

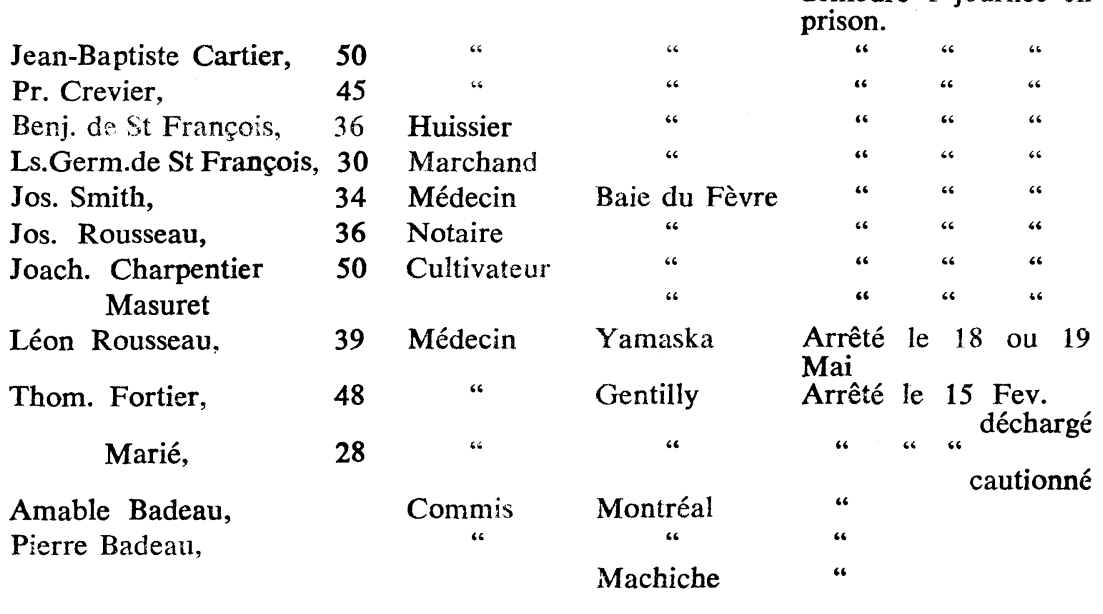




\section{LISTE}

des patriotes qui ont été forcés de fuir du pays ou de se cacher, pour se soustraire à la persécution, depuis la fin de l'année 1837, et contre plusieurs desquels des mandats d'arrêt pour haute trahison avaient été lancés.

Noms

Ls.Jos. Papineau

Edouard E. Rodier

Ludger Duvernay

E.B. O'Callaghan

L.H. Lafontaine

Robert Nelson

\section{Ages Qualifications}

51 M.P.P.

34

38

“ “ "

38

38

32

44

4. 46

46 66

66 66 6

46 86 of
Jn. Ls. Beaudry

Guilln $^{n}$ Beaudreaux

E. R. Fabre

Louis Perrault

Augustin Tulloch

Joseph Martel

Pierre Damour

George Cartier

J. Fériol Peltier

Chamilly de Lorimier 28

Chevalier de Lorimier 30

Charles Levesque 22

Harkins

Amédée Papineau

Josuah Bell

Elisée E. Mailhot

Newcomb

Remy Courcelles

T. S. Brown

J. S. Ney-Smith

Théophile Dufort

Fr. Valois

Fr. Malo

J.B.H. Brien

Messire Chartier

Félix Le Maire

Jacob Banelo

Léandre Dumouchel

Pierre Danis

François Danis

Cyprien Danis

Elie Danis

Joseph Major
24

泟

2

Notaire

droit

Et. en droit

Marchand

Et. en droit

Commis

Tailleur

Publiciste

Marchand

Médecin

Aubergiste

Medecin

Curé

Notaire

Marchand

Médecin

Cultivat ${ }^{\mathrm{r}}$

Marchand

Tanneur

Cultivat $^{\mathrm{r}}$

Demeures
Montréal
“"
“
“

56

46

66

4

46

46

4

60

4

46

6

Montréal

i6

6

c6

46

46

6

66

4

66

Pte Claire

Pte aux Trembles

St Martin

St Benoît

Ste Scholastique

“"

“

“

“

“

“

\section{Remarques}

Orateur de la $\mathbf{C}$.

Ass.

Avocat

Imprimeur Ed.

de la Minerve.

Editeur du

"Vindicator"

Avocat

Chirurgien. Avait été emprisonné à Montréal puis relaché sous caution.

Propriétaire du

"Vindicator".

Fils de rOrateur

I.P. 


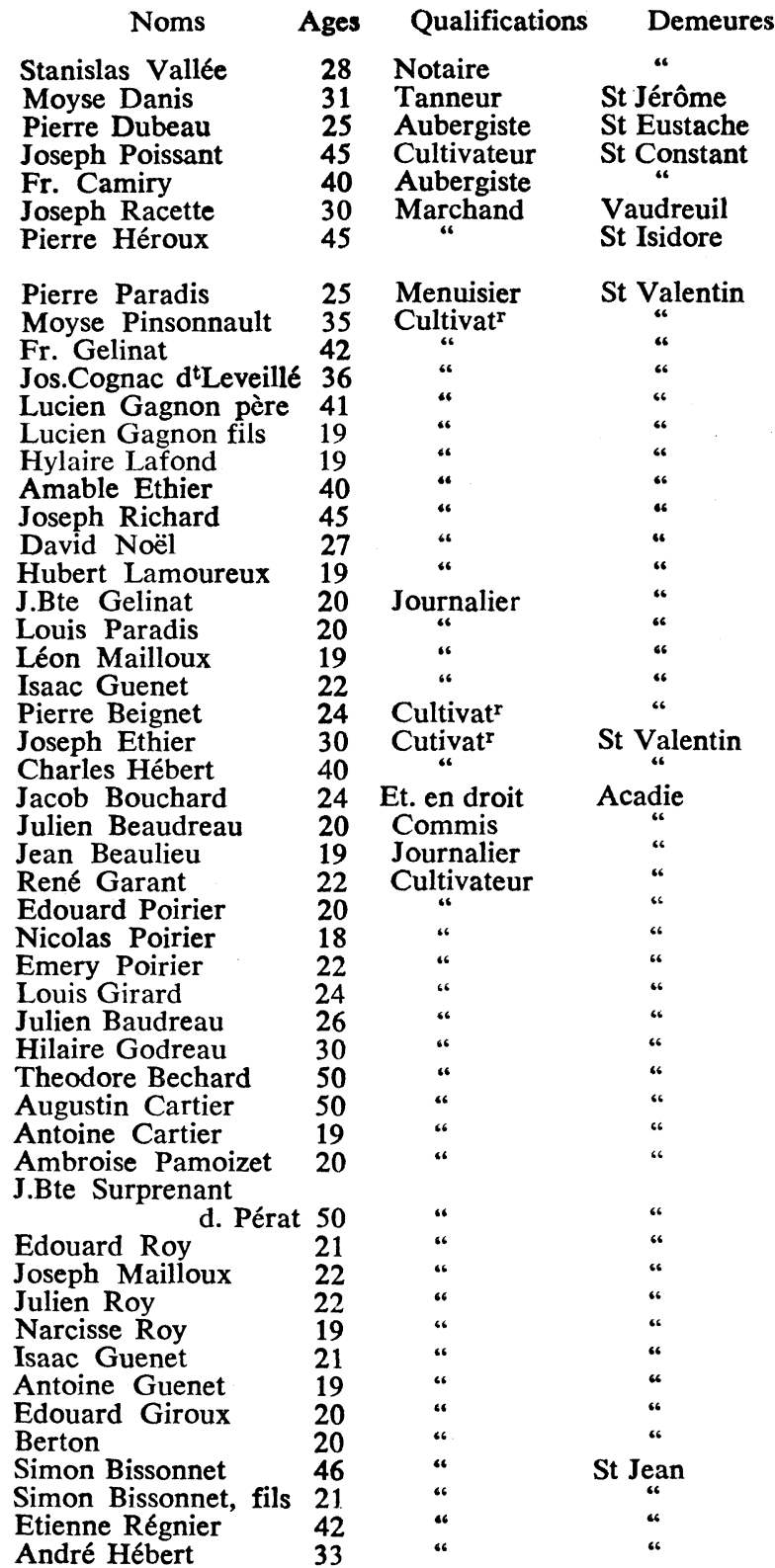

Remarques

Col. M. (Est revenu) 


\begin{tabular}{|c|c|c|c|c|}
\hline Noms & Ages & Qualifications & Demeures & Remarques \\
\hline Jos Davignon & 30 & Médecin & “ & \\
\hline P. P. Desmarais & 40 & Notaire & “ & \\
\hline Edouard Derome & & Forgeron & “ & \\
\hline Edouard Darche & 20 & Commis & “ & \\
\hline Etienne Régnier & 18 & Cultivat ${ }^{r}$ & “ & \\
\hline Douay Ammonean & 22 & Tanneur & “ & \\
\hline Patrick Ammonean & 19 & Cultivat ${ }^{r}$ & “ & \\
\hline Magloire Derome & 23 & Marchand & “ & . \\
\hline Jeremie Sarrazin & 41 & Cultivat $^{r}$ & “ & \\
\hline Joseph Poirier & 19 & Commis & “ & \\
\hline Isaac Laperche & 46 & Menuisier & “ & \\
\hline Gedeon Robert & 38 & Marchand & “ & \\
\hline C. H. Côte & 35 & M.P.P. & Napierreville & Médecin \\
\hline Charles Duquet & 24 & Marchand & La Prairie & \\
\hline Patrick McKiman & 36 & Cultivat $^{\mathrm{r}}$ & St Athanase & \\
\hline Charles Bissonnet & 50 & “ & a & \\
\hline Antoine Robert & 40 & Cultivat ${ }^{r}$ & St-Athanase & \\
\hline Jean Tétro & 24 & Tanneur & St Mathias & \\
\hline Basile Larocque & 30 & Notaire & Chambly & \\
\hline René Boileau & 60 & “ & “ & \\
\hline Joachim Jacques & 34 & Chapelier & St Charles & \\
\hline Henri Cartier & 25 & Médecin & St Antoine & \\
\hline Théodule Gautier & 28 & Peintureur & “ & \\
\hline Isaac Marchessault & 30 & Forgeron & St Ours & \\
\hline Joseph Vincent & 35 & Cultivat ${ }^{r}$ & Longueuil & I.P. Cap. et $\mathbf{M}$. \\
\hline Napoléon Duchênois & 34 & Médecin & Varennes & \\
\hline Thom. Bouthillier & 38 & M.P.P. & St Hyacinthe & Médecin \\
\hline Louis Blanchard & 48 & M.P.P. & “ & Cultivateur \\
\hline Patrice Blanchard & 28 & Arpenteur & “ & \\
\hline Eusèbe Cartier & 47 & Marchand & “ & \\
\hline Fr. Langelier & 27 & “ & “ & \\
\hline J.F. Tétu & 39 & Notaire & “ & \\
\hline Magloire Tuscat & 23 & Et. en Méd. & “ & \\
\hline J. M. Ducharme & 55 & Cultivat ${ }^{r}$ & St Jean-B ${ }^{\text {te }}$ & \\
\hline J.Bte Maillet & 36 & Pottier & St Denis & \\
\hline Joseph Pratte $\operatorname{sen}^{r}$ & 56 & Boulanger & " & \\
\hline Louis Lacasse & 40 & Huissier & “ & \\
\hline Flavien Bouthillier & 35 & Marchand & St Césaire & \\
\hline Fr. Papineau & 61 & Cultivat ${ }^{\mathrm{r}}$ & “ & Major $\mathbf{M}$. \\
\hline Léon Ducharme & 45 & “ & “ & \\
\hline Gaspard Côté & 50 & “ & “ & \\
\hline Joachim Dionne & 30 & $“$ & “ & \\
\hline Joseph Papineau & 26 & Sellier & “ & \\
\hline Joseph Marcoux & 40 & Cultivateur & “. & \\
\hline
\end{tabular}




\section{RÉCAPITULATION}

Membres du Parlement Provincial dont 3 sont avocats, 3 médecins, 1 cultivateur, 1 Imprim., 1 Edit.

\section{Avocats}

Médecins

Prêtres

Publicistes

Notaires

Arpenteurs

Marchands

Etudiants en droit \& en médecine

Libraire

Imprimeur

Artisans

Huissiers

Aubergistes

Cultivateurs \& autres

\begin{tabular}{lr} 
& 9 \\
& 3 \\
& 7 \\
& 1 \\
& 1 \\
& 6 \\
& 1 \\
& 19 \\
& 5 \\
& 1 \\
Total & 1 \\
& 18 \\
& 1 \\
& 59 \\
& 59 \\
\cline { 2 - 2 }
\end{tabular}

Parmi les personnes ci-dessus, il peut y en avoir qui soient revenues et se soient montrées dans la province hors notre connaissance ou pendant que nous rédigions ce Tableau. Un grand nombre d'autres citoyens ont été obligés de laisser la province sous les mêmes circonstances mais nous n'avons pu nous procurer leurs noms. On suppose que le nombre de patriotes que les événemens politiques ont forcés de se tenir cachés ou de se réfugier aux Etats-Unis ne se monte pas à moins de quatre à cinq cents.

\section{LISTE}

des Citoyens, prévenus de haute trahison, pour l'arrestation desquels des recompenses ont été offertes par S. E. Lord Gosford dans ses proclamations.

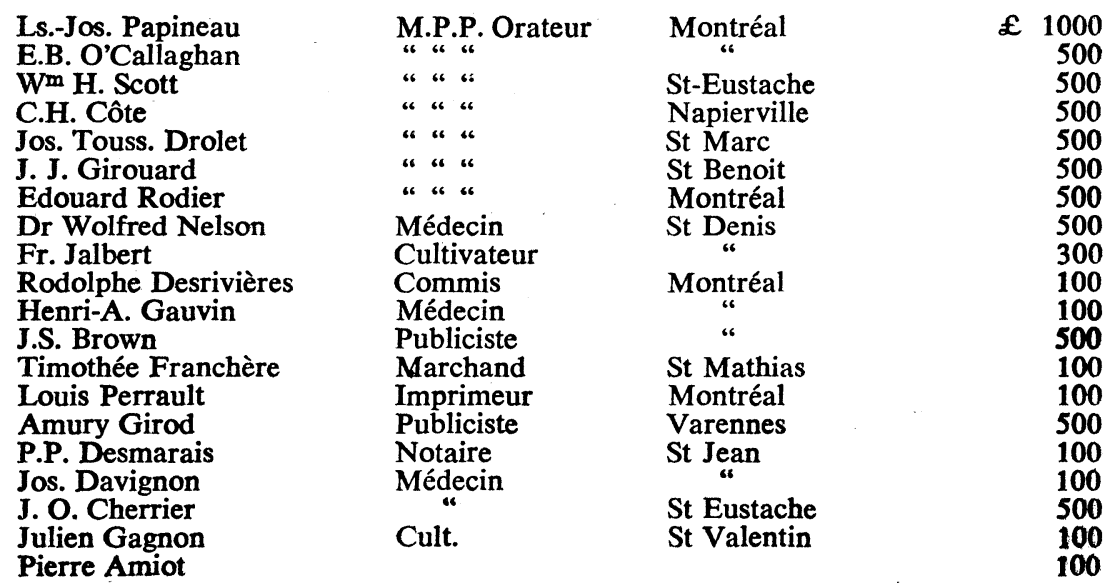




\section{OBSERVATIONS}

PREMIERE. Nous prions les patriotes qui verront ces Tableaux de nous pardonner quelques inexactitudes qui ont pu se glisser dans les noms et les dates, sur lesquels nous avons été dans l'impossibilité de nous procurer des renseignemens certains n'ayant pu d'ailleurs nous aider de documens officiels. Nous pensons cependant que ces erreurs inévitables sont en très-petit nombre.

DEUXIEME. Parmi les patriotes qui ont été incarcérés il y en a plus de cinquante dont les propriétés ont été pillées ou brûlées par les Troupes régulières et les Volontaires de Sa Majesté, dans les expéditions faites au Sud \& au Nord du district de Montréal, sous les ordres immédiats des Colonels Gore, Wetherall \& Maitland, et de Sir John Colborne.

Les pertes éprouvées par les habitans Canadiens qui ont été la victime du despotisme et de la persécution militaire et principalement de la vengeance et de la rapacité des Volontaires et des soit-disant Loyaux, est immense. Un état détaillé de ces pertes, dont la récapitulation a été publiée, en donne un faible apperçu dont voici un extrait.

\section{DANS LE NORD DU DISTRICT}

2 Eglises

2 Presbytères

1 Couvent

1 Moulin à farine

111 Maisons habitées

12 Granges

168 Autres bâtimens

297 Total des bâtimens incendiés

$\begin{array}{cc}\text { Incendiées } & \text { évaluées } \\ \text { “" } & \text { “" } \\ \text { “" } & \text { “" } \\ & \text { “" }\end{array}$

$£ 11500$

1250

450

500

16462

937

1560

Pillage d'environ 575 familles, qui ont perdu en animaux, grains, ustenciles aratoires, meubles de ménage, marchandises, \&c. un montant estimé à

Total

$£ 57556$

\section{DANS LE SUD DU DISTRICT}

A St Denis 45 batimens, à St Charles, 18 bâtimens, pillage de marchandises, animaux, ménages \& autres effets

Total général

Ces estimations ne comprennent aucunement les dommages incalculables résultant de la perte d'un nombre considérable de livres de commerce, titres de familles et de propriétés \&c. Plusieurs greffes de notaires paraissent avoir aussi été brûlés ou dispersés. On ne sait pas encore ce qu'est devenu celui de $M$. Girouard, notaire à St Benoit avec plus de dix mille pièces appartenant aux habitans de cet endroit et des environs. Les Troupes et les Volontaires ont aussi pillé et dispersé la bibliothèque choisie de ce monsieur dans laquelle se trouvaient presque tous les journaux de nos Chambres Législatives, et, en volumes reliés, les principales gazettes publiées en cette Province depuis un grand nombre d'années, en outre l'Histoire du Canada, manuscrite, par feu le Dr Labrie, ouvrage considérable et auquel il avait travaillé toute sa vie; enfin une collection nombreuse et précieuse de pamflets, journaux, notes et autres documents rares sur l'histoire politique et ecclésiastique du pays.

TROISIEME. Les prisonniers politiques à Montréal furent d'abord, pour la plupart, mis dans les cachots. On les y entassa tellement qu'il s'en est trouvé jusqu'à quatre dans un seul cachot de $81 / 2$ pieds sur $51 / 2$. On mit ensuite les nouveaux arrivés dans un quartier destiné aux débiteurs, dans la chapelle et dans quelques uns des appartemens destinés au géolier. Dans la chapelle, formée seule- 
ment de l'attique du centre de la nouvelle prison de Montréal, on a vu entassées jusqu'à quatre-vingt dix-huit personnes, de même que presque tous les autres prisonniers politiques, pendant plusieurs mois, sans lit ni couverture, dans la saison la plus rigoureuse de l'année, sans presque aucun moyen d'entretenir la propreté et de renouveller l'air, manquant même souvent d'eau. Les prisonniers n'ont eu pour toute allouance qu'une livre et demi de pain par jour, encore cette insuffisante pitance dont ceraains détenus avaient à peine de quoi se nourrir la moitié du temps, a-t-elle été souvent diminuée, car sur des plaintes réitérées de la part des prisonniers il fut pesé nombre de pains en présence du shériff, et ils furent tous trouvés légers, la plupart ayant de deux à six onces moins que le poid requis. On dit que le boulanger de la prison en fut quitte en rejettant la faute sur ses garçons, et en envoyant pour cette fois quelques tranches de pain pour parfaire le poid. Les prisonniers ont toujours été privés du droit de voir leurs parens \& leurs amis, et longtemps de communiquer même par lettre. La permission d'aller prendre l'air dans la cour de la prison accordée à tous les autres détenus, ne leur a été commune, et encore avec des restrictions qui la rendent à peu près illusoire, que le 29 Mai dernier. (Lettres et plaintes réitérées au sheriff à ce sujet de la part de plusieurs prisonniers, réponses dérisoires \& cruelles de ce fonctionnaire; son empressement, après la cessation de la loi militaire, à révoquer, à l'égard des prisonniers politiques, par un ordre qu'il a fait homologuer par les Juges de la Cour du Banc du Roi, l'article $8^{\text {me }}$ des Règlemens de la prison qui permet aux prisonniers de voir leurs amis, \& c.)

\section{J. P. Boucher Belleville \& autres *}

\section{N O T E S}

(a) Ce monsieur a d'abord été détenu pendant plusieurs jours dans les cachots (backholes) de l'Isle aux Noix avant d'être conduit à Montréal, avec M.M. $\mathrm{D}^{\mathrm{r}}$ Kimber, Desrivières, Simeon Marchesseau, Cap. Jalbert, J.B. Languedoc, Ls. Dragon, Alex ${ }^{d}$ Noël, Fr. Lafond, Alex. Pinsonnault, Amb. Hébert, Denis Duchêne, Benonie Regnier, Louis Regnier, Julien Tremblay, Ls. Tremblay, Touss. Dufresne, Louis \& Pr. Languedoc, Alex. Richard, Jos. Faneuf, Constant Cartier. Ces infortunés prisonniers furent dans ces cachots, obscurs et infects, sans feu et sans aucune couverture, et ne purent, malgré les plus vives instances, obtenir un peu de paille ou de foin pour se coucher. Il est impossible de dire ce qu'ils eurent à souffrir en cette occasion de mauvais traitemens et de privations de tout genre. Le 11 de décembre ils furent conduits à St Jean, les bras liés derrière le dos, puis liés encore deux à deux par un long câble et si pressés qu'à peine pouvaient ils marcher. On leur fit passer une nuit affreuse au corps de garde de St Jean sans les détacher. M. Bouchette, prisonnier avec eux, en conséquence d'une blessure qu'il avait recue au pied, fut un peu moins maltraité que ses compagnons à l'Isle aux Noix. Le jeune Constant Cartier, blessé à une main, n'éprouva aucune indulgence, et fut fort négligé du chirurgien. Tout le monde connait la manière brutale dont $\mathbf{M}$. Lemaître fut arrêté, et les mauvais traitemens et les cruautés exercés sur un grand nombre de prisonniers, quelques canadiens portent encore la marque des fers dont la rage loyale les ont inutilement chargés pour les conduire en prison.

\section{Dr Lacroix, A.E. Bardy, Le Maître, M.M. Decoigne \& Ranger * \\ M. Berthelot *}

(bb) Ces messieurs, son compagnon de chambre dans la prison M. Ranger, ont essuyé de graves maladies pendant leur détention. Ils exposèrent aux autorités compétentes, et entr'autres à M. de St Ours, par une lettre datée du 8 Mai dernier, combien il leur était difficile de se rétablir dans un lieu comme celui-ci, ne pouvant y recevoir que peu de soins, et pouvant à peine y reposer, à cause du

\footnotetext{
* En marge dans le texte. NDLR.
} 
grand bruit causé continuellement par le rassemblement dans un même local d'un si grand nombre de personnes. Ils demandaient leur élargissement en offrant des cautionnemens au montant qu'on exigerait. Tout cela inutilement. Ils ne purent voir aucun de leurs parens et de leurs amis du dehors et pas même leurs dames qui étaient parties en toute hâte de chez elles pour les venir voir; mais elles n'y purent réussir quoi qu'elles se fussent adressées aux autorités. Je crois que nous étions encore à cette époque sous la loi martiale; mais depuis ce temps M. Berthelot tomba gravement malade, et son fils, avocat de cette ville, s'adressa inutilement au médecin de la prison pour voir son père et le faire sortir. Le Dr Arnoldi le renvoya au procureur général alors à Québec. Celui-ci lui dit de s'adresser au Médecin de la prison qui lui dit que le procureur général lui avait donné ordre de ne donner des certificats aux détenus politiques pour leur permettre de sortir de prison, que lorsqu'ils seraient en danger de mort.

Le Dr Masson*

Longpré *

Le $D^{r}$ Masson, autre détenu, écrivit, le 13 \& le 21 Avril dernier, au secrétaire civil deux lettres des plus pressantes pour obtenir de Son Excellence la permission d'aller donner ses soins, comme parent et comme médecin, à une de ses sœurs aux portes de la mort, offrant de fournir des cautions, et même de payer les dépenses d'une garde qu'on lui donnerait. Sa mère, seul support de cette demoiselle, avait été entièrement ruinée par les Troupes et les Volontaires qui avaient pillé et incendié ses propriétés dans l'affreuse dévastation de St Benoit. Cette jeune personne, ainsi que les autres dames du Village St Benoit, dépouillées par les pillards d'une partie de leurs vètemens, avaient été chassées de chez elles en cet état. Le froid qu'elle endura dans sa fuite était la cause de sa maladie, car ces infortunées dames furent obligées de marcher de nuit pour trouver un gîte, les volontaires ayant menacé de brûler les propriétés de ceux qui leur donneraient asile. Mad ${ }^{11 \mathrm{e}}$ Ovide Le Maire, nièce de M.M. Dumouchel \& Girouard, est morte depuis des suites de la misère qu'elle endura alors. Les lettres du Dr Masson sont restées sans réponse, ainsi que celle adressée au même secrétaire civil par $\mathbf{M}$. Jérôme Longpré de St Jérôme, exposant à S.E. que tous ses enfans sont attaqués de la petite vérole et que deux en restent infirmes; que sa femme elle-même est au lit dangereusement malade, et qu'elle souffre sous le poid de la misère, vû que le seul fils capable de travailler et de la secourir est incarcéré avec son père: c'est en vain qu'il fait la même demande que le Dr Masson.

\section{M.M. Cherrier, Lyonnais, Valois *}

Cependant C.S. Cherrier, M.P.P. le Dr Lyonnais et M. Narcisse Valois, dangereusement malades, purent sortir de prison après bien des difficultés, pour aller se faire soigner ailleurs. Je n'ai pas eu de nouvelles de M. Valois, mais le Dr Lyonnais est mort, et M. Cherrier est encore dans un état alarmant.

\section{Lafond *}

\section{T. Dufresne *}

(c) Son fils, en revenant des Etats-Unis, fut pris à Odelton par les Volontaires qui l'accablèrent de coups. Il se mit alors à faire le fou. Cependant ils lui mirent une corde au cou, le traînèrent à peu près trois arpens et furent près de l'étrangler. Ensuite ils le conduisirent à Richard McGinnis et au Capt. W. Fisher, à Odelton qui le renvoyèrent comme fou. Toussaint Dufresne de St Jean, pris par les Volontaires, fut également menacé d'être pendu, on lui mit la corde au col. Ils firent de plus mine de vouloir le fusiller, tout cela pour l'obliger à dépôser contre quelques-uns de ses concitoyens, ce qu'il refusa néanmoins de faire ainsi que plusieurs autres patriotes contre lesquels les Volontaires et soit disant Loyaux

\footnotetext{
Ces noms en marge dans le texte. NDLR.
} 
ont employé toutes sortes de rigueurs dans la vue d'en faire de vils délateurs. (Voyez plus bas) A St. Benoît.

\section{J.B. Molleur *}

(cc) Des Volontaires, sous la conduite d'un nommé Forbes, allèrent le prendre chez lui. Les ayant vu venir, il descendit par une fenêtre opposée et gagnait un bois voisin, lorsque l'un d'eux fit feu sur lui et le perça d'une balle qui entra près de l'omoplate et sortit près l'artère carotide. Ils le traînerent chez lui où ils poussèrent la barbarie jusqu'à empècher ses amis d'aller lui porter secours, et à repousser à coups de sâbre sa femme qui voulait lui donner ses soins. Il ne dut qu'à l'humanité de M. McGillivray, de Henriville, d'avoir un chirurgien pour panser sa blessure et de rester quelques jours chez lui avant d'être conduit en prison. Il y souffre considérablement de sa blessure. On dit qu'il n'est pas encore hors de danger et que pour le moins il en demeurera infirme toute sa vie.

\section{Lérigé *}

(d) Il n'avait pris aucune part aux événemens politiques, et pourtant on trouva à propos de lâcher contre lui des Volontaires. Ils arrivèrent pendant la nuit à sa demeure, et il se leva pour se remettre entre leurs mains. Sa femme, sur le point d'accoucher, effrayée d'une visite nocturne de la part de tels gens, le pressa à aller se cacher dans la câve. C'est là que les Loyaux le trouvèrent \& le percèrent, quoiqu'il ne fít aucune résistance, de cinq coups de bayonnettes dont un a failli terminer ses jours en prison. Car il est aussitôt lié \& traîné en prison. Sa femme le suit et veut au moins pouvoir étancher le sang qui coule de ses blessures, mais ni ses prières, ni ses larmes, ni ses cris déchirans, n'empèchent ces barbares de la repousser à coups de pieds et de bayonnettes, la menaçant même de faire feu sur elle si elle ne se retire.

\section{Fr. Renaud *}

(e) Les privations et les souffrances d'un cachot sale et mal aéré où il n'avait ni lit, ni couverture, ni nourriture suffisante, avaient presque entiérement détruit sa santé. Il demanda au Médecin de la prison à être transporté à l'infirmerie. Ses prières furent longtemps et plusieurs fois repoussées, lorsqu'un rhumatisme inflammatoire dont il souffrait, prit un caractère tellement inquiétant que le Dr Arnoldi se vit forcé d'acquiescer à sa demande. Il fut plusieurs jours dans un état désespéré; cependant l'habileté et les secours opportuns du Dr W. Nelson, joints aux soins empressés de ses autres compagnons d'infortune, l'ont rappelé à la vie. On pourrait peut-être ajouter que sans les secours du même Dr Nelson, plusieurs prisonniers auraient trouvé la mort dans leur dongeon.

\section{Marineau *}

(f) L'enfant d'un de nos concitoyens de ce nom à St Eustache, âgé de 10 à 11 ans, fut tué par un soldat d'un corps des Volontaires qui, peu après l'affaire de St Eustache, parcouraient la campagne, fesaient toutes sortes de violences aux pauvres habitans canadiens, et commettaient impunément toutes sortes de vexations et de déprédations. Un motif de curiosité avait fait sortir cet enfant de sa maison avec quelques autres membres de sa famille. Le Volontaire tira son pistolet dans le groupe \& l'enfant tomba mort. Le Volontaire a été détenu quelques jours en prison d'où il est sorti sans caution ! Un autre Loyal sujet de S.M. en a aussi été quitte à bon marché. M. François Plante, riche propriétaire de la Prairie, a été fusillé tandis qu'il était paisiblement occupé dans la cour de sa maison. L'assassin n'a pas été inquiété; il lui a suffi de dire aux autorités qu'il ne l'avait pas fait exprès.

\footnotetext{
* Ces noms en marge dans le texte. NDLR.
} 


\section{Th. Roy *}

(g) Vers le 25 Novembre les Volontaires de St Jean, sous le commandement de George Mc Donald, entrèrent chez lui pour le faire prisonnier. Ne le trouvant pas ils sortirent pour aller briser les bacs et les canots des traverses voisines. L'un d'eux (dont j'ai pardevers moi le nom avec des déclarations) resté seul auprès de madame Roy, ferme sur lui la porte et ôse faire des propositions infâmes à cette dame, qui aurait peut être été la victime de sa brutalité s'il n'était tout à coup arrivé quelqu'un au logis. J'aurais à rapporter bien d'autres traits de ce genre si j'avais voulu les recueillir.

\section{J.J. Girouard et autres *}

(h) Il partit de chez lui lors du sac de St Benoit, où tout ce qu'il possédait fut pillé et brûlé par les Troupes et les Volontaires, sous les ordres immédiats de Sir John Colborne. Ce Monsieur s'était refugié dans le comté de Vaudreuil et attendait dans une retraite sûre depuis plusieurs jours que les glaces pussent lui permettre d'effectuer son passage de l'autre côté de la ligne, par un moyen certain que des amis lui procuraient. Cependant ayant appris que les prisons de Montréal se remplissaient tous les jours de ses alliés et de ses amis, il changea d'avis \& écrivit au $\mathrm{L}^{t}$ Colonel Simpson: qu'il avait entendu (....mot manque) n'avait point vu les journaux, que Lord (... mot manque) avait mis sa tête à prix; que sa con (... mot manque) reprochait rien dans sa conduite et comme (... mot manque) public et comme simple citoyen, que néanmoins il avait dû se soustraire aux passions (... mot manque) et des barbares qui avaient parcouru (...mot manque) son comté. Qu'il n'était pas en fuite (...mot manque) voulait partager le sort de ceux de ces (... mot manque) avaient honorablement suivi la même conduite que lui; mais qu'il ne voulait pas s'exposer à devenir le jouet ou la victime des prétendus Loyaux et de ces êtres vils qu'une indigne récompence encourageait à commettre le crîme. Qu'il n'avait aucune objection à se remettre entre les mains d'un homme d'honneur comme lui (M. Simpson) sur qu'avec sa protection, il ne lui serait fait aucune insulte. En effet M. Simpson eût la complaisance de se rendre lui-même à la retraite de $M$. Girouard qu'il conduisit jusque dans les prisons de Montréal, dans une voiture à quatre chevaux bien couverte, accompagné de deux amis bien armés, en cas de surprise. Grâce aux soins généreux de M. Simpson, M. Girouard put donc rejoindre ses amis en prison, sans être exposé aux joies brutales et aux insolences des Constitutionnels. Nous aimons à publier ce fait à la louange de M. Simpson qui, comme magistrat a encore d'autres titres à notre reconnaissance; car nous avons appris que la conduite prudente et généreuse qu'il avait tenue dans son endroit à l'égard des canadiens persécutés lui avait attiré bien des déboires et qu'on avait même été jusqu'à l'accuser auprès du gouverneur.**

M.M. Lacoste M.P.P. Drolet père, M.P.P., le Dr Duvert, Franchère, Marchand, Gigon, Mongeon, Loupras \& Knight M.P.P. qui avaient appris que des ordres d'arrestation étaient émanés contre eux, et poussés sans doute par les mêmes motifs que $M$. Girouard, se constituèrent volontairement prisonniers. Les six derniers Messieurs s'étaient rendus jusqu'à Highgate $V^{t}$.

\section{Messire Blanchet *}

(i) A l'arivée de ce monsieur en prison le Sheriff n'eut pas honte de venir lui reprocher sa conduite, et de se permettre de lui faire des réprimandes les plus déplacées comme les plus injurieuses. Plusieurs autres prisonniers ont eu à se plaindre de ce que ce fonctionnaire aît eu la cruauté d'insulter pour ainsi dire à leur malheur, en leur adressant des mercuriales sans doute cette espèce de punition infligée aux détenus n'avait pas été ordonnée. Ceci ne doit

* Ces noms en marge dans le texte.

* Les "quelques mots qui manquent" sont dus à une déchirure de la page. 
pas étonner de la part de cet homme auquel les prisonniers doivent en grande partie les mille et une privations et restrictions dont ils ont eu à gémir pendant deux longues détentions. Ne l'ai-je pas entendu moi-même justifier les horreurs dont les Troupes et les Volontaires se sont rendus coupables, en rejetter la faute sur ses compatriotes, et aller jusqu'à dire que le carnage, les incendies et le pillage fait dans le sud et le Nord du district étaient des actes nécessaires, qu'il fallait faire un exemple, \&c. Pour revenir à Messire Blanchet, le Canadien ayant publié une communication dans laquelle on rapporte quelques unes des déprédations et des attrocités commises à St Denis et à St Charles par les vainqueurs, à la honte de l'armée anglaise, le même $\mathbf{M}$. de St Ours vint avec deux officiers de l'Etat Major, trouver Messire Blanchet, et fit son possible pour l'engager à donner à ces officiers un certificat démentant les allégués du Canadien; ce que M. Blanchet refusa de faire alléguant qu'il ne pouvait commencer par contredire l'article du Canadien qui, en autant que les choses étaient venues à sa connaissance, ne contenait que la vérité. Il nous est aussi agréable de citer ce trait à l'honneur de Messire Blanchet, qu'il nous est pénible d'avoir à dévoiler la conduite honteuse d'un compatriote qui au lieu de consoler et protéger, comme il le pouvait ses frères persécutés, et souffrans, s'est fait le très humble serviteur et le vil et complaisant instrument de la tyrannie.

\section{Larocques *}

(k) M. Larocques a été emprisonné pour avoir fait réimprimer à Montréal un article du London Westminster Review en faveur des Canadiens dont l'impression en Angleterre n'avait fait emprisonner ni les éditeurs, ni les imprimeurs. En Canada l'édition a été saisie par autorité de Justice avec les types et les presses, l'imprimerie qui a servi à son impression et tout demeure encore sous saisie.

Montréal 1 Juin 1838

A ajouter à la note C. Lafond.) A St Benoit, lorsque Sir John Colborne était ... (quelques mots manquent) ses militaires accompagnés d'in ... (quelques mots manquent) Loyaux, il n'est point de menaces et de... (quelques mots manquent) que l'on ne fit à plusieurs habitations ... (quelques mots manquent) à découvrir la retraite de ceux ... (quelques mots manquent) Deux canons purent ... (le reste de la phrase est illisible). (Deux autres lignes sont illisibles). Brazeau fut étendu plusieurs fois ... (quelques mots manquent) l'on se mit en devoir de ... (quelques mots manquent) des indices de la retraite de $\mathbf{M}$. Girouard ... conduit la veille jusqu'aux ... (quelques mots manquent) honneur de tous en dignes patriotes qu'ils étaient ... (quelques mots manquent) et que les promesses et les récompenses qui leur ont été offert n'eussent pas plus d'effet.**

Tandis que M. Lafond père était... (mot illisible) c.a.d. vers le mois de Janvier dernier, une dizaine de Volontaires entrèrent dans sa maison à St Valentin sous prétexte d'y chercher des armes. N'en trouvant point ils s'en prirent à Madame Lafond et à sa mère, agée de 77 ans, qu'ils insultèrent de la manière la plus grossière, se portant même à des violences sur les personnes de ces femmes que parce qu'elles voulaient s'opposer à ce que ces vandales emportassent les papiers qu'ils avaient trouvés en fouillant la maison, et parmi lesquels se trouvaient des titres de propriété et autres papiers, outre tous les procès, les baux de voierie dont $M$. Lafond était dépôsitaire comme inspecteur de la paroisse. Tous ces papiers furent déchirés. Madme Lafond fut porter ses plaintes à St Jean chez le Juge de Paix Mc Rea auquel elle montra les marques qu'elle portait encore sur les bras de la violence des Volontaires. Il se contenta de lui dire: Votre mari est un rebelle; c'est bon pour vous.

\footnotetext{
* Ces noms en marge dans le texte.

** Note de la copiste: Les "quelques mots qui manquent" sont dus à une déchirure de la page.
} 